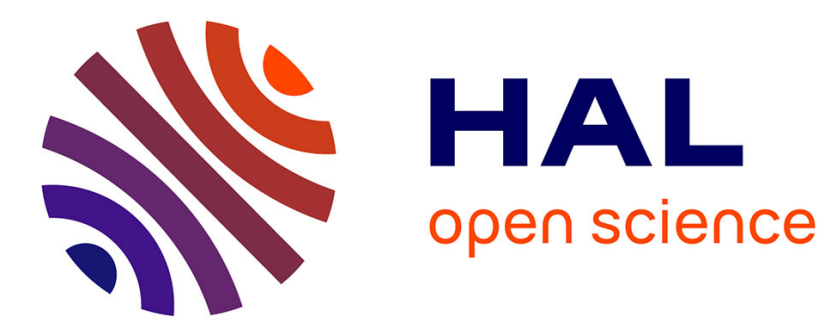

\title{
Magnetic Nanoparticles: From Synthesis to Theranostic Applications
}

\author{
Sumera Khizar, Nasir Ahmad, Nadia Zine, Nicole Jaffrezic-Renault, \\ Abdelhamid Errachid, Abdelhamid Elaissari
}

\section{- To cite this version:}

Sumera Khizar, Nasir Ahmad, Nadia Zine, Nicole Jaffrezic-Renault, Abdelhamid Errachid, et al.. Magnetic Nanoparticles: From Synthesis to Theranostic Applications. ACS Applied Nano Materials, 2021, 4 (5), pp.4284-4306. 10.1021/acsanm.1c00852 . hal-03244150

\section{HAL Id: hal-03244150 \\ https://hal.science/hal-03244150}

Submitted on 1 Jun 2021

HAL is a multi-disciplinary open access archive for the deposit and dissemination of scientific research documents, whether they are published or not. The documents may come from teaching and research institutions in France or abroad, or from public or private research centers.
L'archive ouverte pluridisciplinaire HAL, est destinée au dépôt et à la diffusion de documents scientifiques de niveau recherche, publiés ou non, émanant des établissements d'enseignement et de recherche français ou étrangers, des laboratoires publics ou privés. 


\title{
Magnetic Nanoparticles: From Synthesis To Theranostic Applications
}

Sumera Khizar, Nasir M. Ahmad*, Nadia Zine, Nicole Jaffrezic-Renault, Abdelhamid Errachidel-salhi, and Abdelhamid Elaissari*

Polymer Research Lab, School of Chemical and Materials Engineering (SCME), National University of Sciences and Technology (NUST), H-12 Sector, Islamabad-44000, Pakistan Univ Lyon, University Claude Bernard Lyon-1, CNRS, ISA-UMR 5280, F-69622 Lyon, France

\begin{abstract}
Over the past few years, engineered inorganic nanoparticles have been studied researched, and applied extensively in the biomedical field since resultant platforms increase the usage of the nanoparticles for particular theranostic applications. The innovation of such nanosystems has the potential to develop a theranostic mode that integrates both diagnosis and treatment of diseases in a particular system via combinatorial approaches of imaging, targeting, and therapy that accomplish the potential of personalized and tailored medicine. The manipulation of magnetic nanoparticles (MNPs) as theranostic agents have attained growing consideration in material science owing to their exclusive capability in magnetic targeting, magnetic resonance imaging, chemotherapy, hyperthermia, bioseparation, gene therapy, enzyme immobilization, and controlled release of the drug. To intensify the diagnostic and therapeutic efficacy, MNPs have been ornamented or functionalized with a range of materials to enhance the biocompatibility, stability, and capability to carry therapeutic payloads and to encapsulate imaging agents. Preferentially synthetic and natural polymers have been exploited to coat for ensuring their colloidal stability and good dispersibility in biological fluids. Stimuli-responsive polymers have also been used to functionalize MNPs to establish a therapeutic approach that can significantly enhance therapeutic effectiveness including the real-time monitoring in specific cells and tissues. The present review highlights the progress of MNPs emphasizing functionalization using diverse inorganic and organic and biomaterials. Furthermore, it also provides the soundproof concept on the potential of MNPs and their colloidal counterparts that offer attractive possibilities for theranostic applications. Finally, the main challenges and limitations in the use of MNPs for advanced biomedical applications have also been critically provided.
\end{abstract}


KEYWORDS: magnetic nanoparticles, theranostics, nanovectors, MRI, hyperthermia, chemotherapy, bioseparation, photothermal

\section{INTRODUCTION}

At present nanotechnology is mostly applicable in biomedical applications due to the recent advances in the understanding of materials combined with other materials that can safely be incorporated for medical diagnosis and therapeutics. Nanoparticles offer several beneficial experiences for numerous bioapplications on account of their exceptional chemical, biological, and physical properties. Nanoparticles composition (lipids, metals, peptides, polymers) and shapes (spheres, pyramids, rods, and flowers) are influenced by the synthesis methods and functionalization procedures. The conception of the operative nanosystem aimed at theranostic nanoparticles trying out new medication which at the same time provides together imaging and therapeutic agents for disease management. ${ }^{1}$ The blend of imaging and therapeutics permits huge handling of treatment, currently named theranostics that describes single and biocompatible nanoparticle which can be used in both diagnostic and therapeutic. Theranostic is the integration of multiple moieties into a single nanoplatform for diagnostic and therapeutic purposes. A large amount of work is dedicated to biomedical uses as conventional nanoparticle systems distinctly. However, several administrations are essential to achieve all vital functions that bring apprehensions about persistent compliance of patient and safety with care. For theranostics to be effective, multifunctional preparations which consist of nanoparticle used for drug transfer with incorporated contrast agents with fewer side effects related to old-style chemotherapy procedures. A new trend is developing for using MNPs for theranostic uses due to its biocompatibility, sizes, the capability of biofunctionalization, and their responses to the external field. $^{2}$

Three main approaches include the usage of nanoparticles in theranostic applications. The first approach involves treatment with effective estimation via imaging at the molecular level; the second approach describes molecular imaging probes to assess the therapeutic strategy of nanoparticles. The third one defines nanoparticles as a tool for imaging and therapy at the molecular level at the same time. ${ }^{3}$ By integrating diagnosis into therapy, theranostic approaches are beneficial for planning and follow-up of treatment and monitoring of objective response centred on characteristics of molecular disease. ${ }^{4}$ The developing integrated strategies 
revolutionize the theranostic systems to assess the efficacy of nanoparticles.

In this respect, magnetic theranostic nanoparticles are of noticeable importance and have been studied, investigated, and applied widely to biomedical applications for diagnosis and therapy For biomedical applications, these days nanotechnology has enhanced the features and designs, explicitly the essential requirements and properties of MNPs. They have increased popularity and emerged as important biomedical functional nanomaterials because of their exceptional characteristics such as chemical stability, biocompatibility, high saturation magnetization, and less toxicity and capacity to work at the molecular and cellular level. ${ }^{5}$ MNPs can be used for diagnosis, gene delivery, hyperthermia, drug delivery, phototherapy, bioseparation, imaging mechanisms, chemotherapy due to their inimitable characteristics and can be used in combinational approaches for theranostics. ${ }^{6,7} \mathrm{~A}$ wide variety of nanomaterials have been employed for better contrast agents encapsulation, biocompatibility, transportation of therapeutic payloads, and to provide functional groups to conjugate biomolecules to enhance the efficiency of MNPs, that are used for theranostic applications. A significant effort has been dedicated to the synthesis and design of MNPs that are ferromagnetic with controlled parameters. 5,8

Mostly MNPs used in the biomedical application are made of nanoparticles of iron oxide (IONPs e.g. $\gamma-\mathrm{Fe}_{2} \mathrm{O}_{3}$ (maghemite) or $\mathrm{Fe}_{3} \mathrm{O}_{4}$ (magnetite)) and gadolinium (chelated organic gadolinium complexes) because of their capability of decomposition into oxygen and iron in the body. After degradation, they are used in oxygen transport and metabolic systems and can easily be removed from the body. When nanoparticles are fabricated of size of approximately $10 \mathrm{~nm}$ in diameter, IONPs show superparamagnetic behaviour (superparamagnetic iron oxide nanoparticles, SPIONs) accountable to better dispersability without magnetic field. ${ }^{9}$ They accumulate at the target site in magnetic field presence that is of immense significance in theranostic uses. ${ }^{10}$ IONPs can be doped by elements having magnetic susceptibility (e.g. $\mathrm{CoFe}_{2} \mathrm{O}_{4}, \mathrm{MnFe}_{2} \mathrm{O}_{4}$, etc.) and metal alloys (e.g. FePt, FeCo, etc. nanoparticles), but they cannot be engaged in medical applications because of their rapid oxidation with potential toxicity. Though, the magnetic properties of these metal alloys and ferrites are robust than that of the pure iron oxide. $^{5}$

For theranostic applications, MNPs must be monodisperse and have a uniform composition. Each distinct nanoparticle possesses nearly indistinguishable chemical and physical 
characteristics for controlled bioelimination, biodistribution, and contrast effects. ${ }^{11}$ Their magnetic moment should also be high and can be altered through bonding precisely to biomolecules of concern. They also should have the capability to resist diverse physical and biological conditions. ${ }^{2}$ Among MNPs, magnetite is relatively a promising material for bioapplications because of its adequate biocompatibility, reduced toxicity, effective magnetic characteristics, and has also been authorized with United States Food and Drug Administration (FDA). ${ }^{12,} 13$ Table 1 displays the record of IONPs approved by the Food and Drug Administration (FDA) and the European Commission (EC) for clinical applications. Later the usage of sinerem and ferumoxide was abandoned. ${ }^{14}$ Only two SPIONs-derived products approved by FDA are commercially available are GastroMARK and ferumoxytol. ${ }^{14,15}$ Less toxicity of nanoparticles was also established through in vivo studies in animals via morphological investigation of internal organs of animals by administrating magnetite nanoparticles by various methods. ${ }^{5}$ MNPs have a pronounced ability to evaluate and predict diagnostic and responses in therapy. Their usage not only provides profitable prospects for transformation and modification of on-going treatments but also beneficial for the development of new tools in personalized manner.

Table 1. List of iron oxide nanoparticles approved and abandoned by FDA for clinical usage. Reproduced from ref ${ }^{14}$. Copyright 2021 PubMed Central.

\begin{tabular}{|c|c|c|c|}
\hline IONPs & Indication & Size & Administr \\
\hline Lumirem, approved by & Contrast agent for MRI for & $50 \mathrm{~nm}$ & Oral \\
\hline FDA & gastrointestinal tract & & \\
\hline $\begin{array}{l}\text { Ferumoxides or Feridex, } \\
\text { approved by FDA, later } \\
\text { abandoned }\end{array}$ & $\begin{array}{l}\text { Contrast agent for MRI for liver } \\
\text { cancer }\end{array}$ & $\begin{array}{l}120-180 \\
\mathrm{~nm}\end{array}$ & $\begin{array}{l}\text { Injectable } \\
\text { solution }\end{array}$ \\
\hline $\begin{array}{l}\text { Ferucarbonate, } \\
\text { approved by FDA }\end{array}$ & Contrast-enhanced MRI of liver & $\begin{array}{l}45-60 \\
\mathrm{~nm}\end{array}$ & $\begin{array}{l}\text { Injectable } \\
\text { solution }\end{array}$ \\
\hline Sinerem, authorized by & Detection and characterization of & $10-40$ & Injectable \\
\hline $\begin{array}{l}\text { European marketing, } \\
\text { later abandoned }\end{array}$ & $\begin{array}{l}\text { metastatic lymph nodes in patients } \\
\text { with pelvic cancer }\end{array}$ & $\mathrm{nm}$ & solution \\
\hline
\end{tabular}




\begin{tabular}{llll}
\hline $\begin{array}{l}\text { Ferumoxtram, approved } \\
\text { by FDA }\end{array}$ & $\begin{array}{l}\text { Detection and characterization of } \\
\text { metastatic lymph nodes in patients } \\
\text { with pelvic cancer }\end{array}$ & $\begin{array}{l}10-40 \\
\mathrm{~nm}\end{array}$ & $\begin{array}{l}\text { Injectable } \\
\text { solution }\end{array}$ \\
$\begin{array}{l}\text { Ferumoxytol, approved } \\
\text { by FDA and EC }\end{array}$ & $\begin{array}{l}\text { Detection and characterization of } \\
\text { metastatic lymph nodes in patients } \\
\text { with pelvic cancer }\end{array}$ & $\begin{array}{l}\text { nm } \\
\text { nm }\end{array}$ & Intravenous \\
\hline
\end{tabular}

\section{NANOVECTORS IN THERANOSTICS}

The area of nanotechnology proposes an amazingly extensive range of innovative and advanced nanovectors with high adaptability and compliance for theranostics that includes both diagnosis and therapy. Nanomaterials with many nanovector-based formulations gave a significant contribution to the development of such theranostic systems. It encloses the handling of particles to work with them at the molecular and subatomic levels to study and treat diseases. The capability of nanovectors to carry and deliver one or more bioactive molecules assists the incapacitating of some traditional diagnostic complications, such as detection of a multiplexed analyte, extraordinary sensitivity, and reproductive signal amplification to quantify biomarkers present in the body fluids at an ultra-low concentration. Nanotechnology provides a platform to design and develop such nanovectors that deliver innovatory stimulation therapy. As a result, the interactiveness of nanoparticles with the selected area of target promotes biological reactions along with the least harmful effects.

Previously magnetic nanoparticles, noble metal nanoparticles, and quantum dots (QDs) were in employment for several diagnostic and therapeutic purposes. ${ }^{16,17}$ Recent developments have created an opportunity to discover novel theranostic nanovectors that can be used in the management of disease to overcome the shortcomings of orthodox nanoparticle systems such as safety and patient compliance. Various designed nanovectors are united with molecular imaging probes and drug comprising inorganic and metal (silver, gold, iron oxide, and silica nanoparticles) nanoparticles, polymer nanoparticles including micelles, liposomes, and dendrimers as shown in Figure 1, defining the best combination for specific theranostic applications with fewer side effects. ${ }^{18}$ 


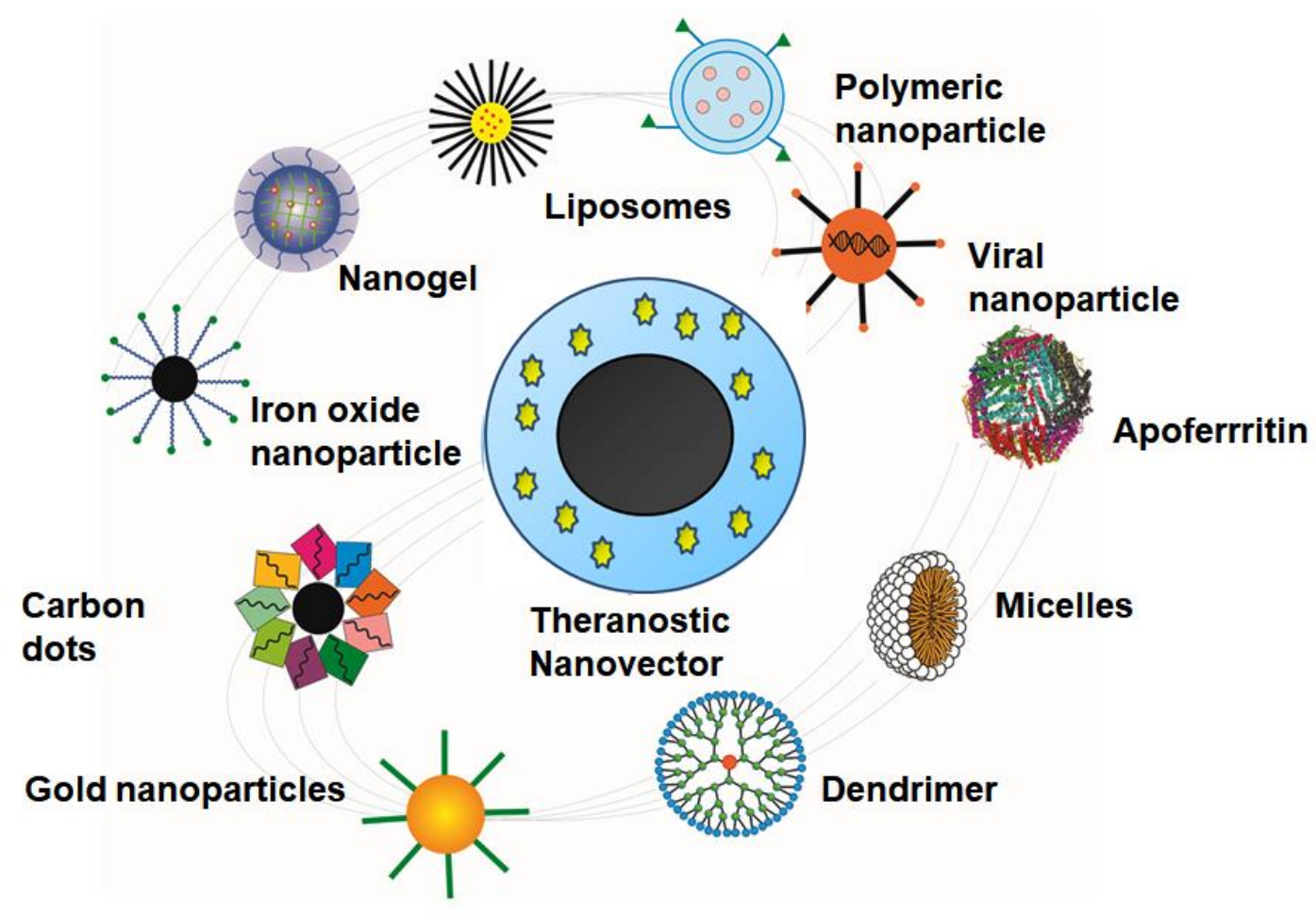

Figure 1. Schematic illustration of nanovectors towards theranostics.

Recently, the interest in using nanoparticles of metal like silver, gold, palladium, platinum, bismuth, zinc, molybdenum, manganese, copper, and iron have been developed in diverse biomedical applications, like biosensors, sustained/target delivery of the drug, photothermal therapy, bioimaging, as well as hyperthermia. ${ }^{19-22}$ The nanoparticle's variation, stabilization, and modification by particular functionalization groups permits the binding of drugs, ligands, and other antibodies to them, which make the structures further favorable theranostic agents. The most fascinating theranostic nanovectors among inorganic nanomaterials are IONPs due to their distinctive physicochemical characteristics at the nanoscale. They generate huge benefits for nanoparticulate structures for the primary and timely recognition and active drug delivery of drugs for improved theranostics approach. ${ }^{23}$ Gold and silver nanoparticles have also been extensively explored as multifunctional nanovectors and exhibit distinctive optical and thermal properties,${ }^{24}$ which can readily upgrade these nanomaterials to be prospective theranostic agents. ${ }^{25,26}$ They are easy to synthesize and functionalize, relatively less toxic, and exhibit numerous benefits such as versatility and biocompatibility. These nanovectors are used as X-ray contrast agents, surface-enhanced Raman spectroscopy (SERS) enablers, and in radiotherapy enhancement. ${ }^{27}$ Polymeric nanoparticles and micelles are also used as probes for 
imaging and carriers for theranostics because of their high drug payload and scalability, sustained release of the drug, low-cost production, low batch variability, easier tenability, and degradation properties. ${ }^{28,29}$ The usage of polymeric micelles is helpful because of their ability to entrap contrast agents and hydrophobic drugs, preserving their hydrophilic characteristics as carriers, compared to soluble polymers or liposomes. ${ }^{30}$ Liposomes, artificially generated vesicles, have essentially been examined as vehicles for delivery of drugs following their favorable invulnerable profile. Multifunctional nanovectors have been created with liposomes encapsulating contrast agents for the instantaneous release of drug and early diagnosis of a disease. ${ }^{31}$ Dendrimers gain increasing significance in the field of theranostic by virtue of their size and encapsulating ability of imaging tracers along with several drugs with high efficacy. The dendrimers as multifunctional nanovectors represent promising structures for theranostics because of excellent biocompatibility, biodistribution, and satisfactory diagnostic and therapeutic efficacy. ${ }^{32}$ Quantum dots (QDs), inorganic semiconductor nanocrystals can be conjugated noncovalently or covalently with probes for targeting, like several folate aptamers, antibodies, nucleic acids, peptides, and small molecules that signify a probable participant for theranostic purposes. They showed clinical potential limitations attributed to their potential toxicity in humans. ${ }^{33}$ The improvement of chemotherapeutic agents by carbon nanomaterials make them valuable for diagnostics and therapeutic applications. But because of subsequent toxicity and slower biodegradation, they had poor clinical potential. ${ }^{34}$ Silica nanoparticles are biodegradable and biocompatible materials and widely used in applications of nano theranostics. They have been thoroughly investigated in diagnostics because of their tunable shape and size, and extraordinary drug loading capacity attributed to the wide surface area. ${ }^{35}$ Due to superior stability, biocompatibility along biodegradability, modern researchers get encouraged to use these bio-nano particles for theranostic research. They include solid lipid nanoparticles, protein nanoparticles, viral nanoparticles, apoferritin, and aptamers, where the bio-mimicking constituent is assimilated into therapeutic nanoparticles. These forms of nanoparticles were effectively synthesized, designed, and employed for theranostics applications. ${ }^{36}$ Table 2 presents a list of various designed nanovectors combined with drug and molecular imaging probes of a certain size.

Table 2. Various nanovectors employed in theranostic applications. 


\begin{tabular}{|c|c|c|c|c|}
\hline Type of nanovectors & Biosafety & Size & Main theranostic Applications & Ref \\
\hline $\begin{array}{l}\text { Metallic Nanoparticles } \\
(\mathrm{Cu}, \mathrm{Pd}, \mathrm{Zn}, \mathrm{Bi}, \mathrm{Mo}, \\
\mathrm{Mn}, \mathrm{Ce}, \mathrm{Pt})\end{array}$ & $\begin{array}{l}\text { Potential } \\
\text { toxicity }\end{array}$ & $2-70 \mathrm{~nm}$ & Delivery vehicle & $17,20,22$ \\
\hline Magnetic & Potential & $10-20 \mathrm{~nm}$ & Drug delivery, MRI, Contrast agent & 23 \\
\hline Nanoparticles & toxicity & & & \\
\hline Gold Nanoparticles & Low toxicity & $10-60 \mathrm{~nm}$ & SERs, Biomedical applications & 37,38 \\
\hline Silver Nanoparticles & Low toxicity & $10-50 \mathrm{~nm}$ & $\begin{array}{l}\text { Biosensors, Drug delivery, Molecular } \\
\text { imaging, In vivo cancer therapy and } \\
\text { diagnosis }\end{array}$ & 39 \\
\hline $\begin{array}{l}\text { Polymeric } \\
\text { Nanoparticles/micelles }\end{array}$ & Low toxicity & $20-70 \mathrm{~nm}$ & $\begin{array}{l}\text { Delivery of water-insoluble drugs, } \\
\text { Nutraceutical delivery systems }\end{array}$ & 28 \\
\hline Liposomes & Less toxicity & $10-70 \mathrm{~nm}$ & Drug delivery, Gene encoding & 31 \\
\hline Dendrimers & $\begin{array}{l}\text { Potential } \\
\text { toxicity }\end{array}$ & $10-40 \mathrm{~nm}$ & $\begin{array}{l}\text { Biomedical applications, Drug carrier, } \\
\text { Imaging agents, Gene delivery }\end{array}$ & 32 \\
\hline Quantum Dots (QDs) & $\begin{array}{l}\text { Potential } \\
\text { toxicity }\end{array}$ & $2-50 \mathrm{~nm}$ & $\begin{array}{l}\text { Drug Delivery, Diagnostics, and } \\
\text { biomedicine }\end{array}$ & 33 \\
\hline $\begin{array}{l}\text { Carbon } \\
\text { Nanomaterials }\end{array}$ & $\begin{array}{l}\text { Potential } \\
\text { toxicity }\end{array}$ & $0.4-40 \mathrm{~nm}$ & Biomedicine & 40 \\
\hline Silica Nanoparticles & Low toxicity & $20-25 \mathrm{~nm}$ & Drug delivery & 41 \\
\hline Bio-Nanoparticles & $\begin{array}{l}\text { Very low } \\
\text { toxicity }\end{array}$ & $20-500 \mathrm{~nm}$ & $\begin{array}{l}\text { Testing and treatment of various } \\
\text { viruses }\end{array}$ & 36 \\
\hline
\end{tabular}

\section{SYNTHESIS OF MAGNETIC NANOPARTICLES}

For the last 20 years, there has been implausible research work to investigate and advance various ways of controlling the physical and chemical properties of IONPs. For basic and 
biomedical benefits, it is a strong need to prepare discrete and distinct MNPs. There are many physical and chemical methods to design MNPs in such a way as to have biocompatible surface stabilizers for biomedical applications. ${ }^{42}$ Considerable efforts have been put into design, development, and improvement that makes highly pure nanomaterials and enables their mass production, but the shape and size of nanoparticles are hard to control. ${ }^{43}$ In very broad terms, there are three distinct methods (physical, chemical, and biological) being employed for the synthesis of MNPs and they are well documented and studied throughout the literature. ${ }^{44}$ To deal with these side effects, numerous chemical techniques have been explored to prepare highquality nanoparticles depending on their colloidal chemistry in the solution-phase.

Synthesis of MNPs via physical methods includes the decomposition of magnetic responsive materials to target the desired site. These methods are difficult because they need high energies, skilled manpower, and optimized sophisticated parameters for their operations. Biological methods are distinctive and highly localized in the sense that they involve magnetosomes in well-defined lipoprotein chambers that prevail inside the living body. ${ }^{45}$ This permits the host to develop a magnetic field inside it that responds to the magnetic field of the earth. Chemical methods are the most versatile and enable excellent control over shape, morphology, and nanoparticle size. They are simple, highly productive, and can be carried out without any complicated equipment. Chemical methods have advantages over the other methods because they are environmentally friendly and can easily scale up for large synthesis of nanoparticles. ${ }^{46}$ The comparison of different chemical methods is summarized in Table 3.

Table 3. Differentiation of various chemical approaches for synthesizing magnetic nanoparticles.

\begin{tabular}{|c|c|c|c|c|c|c|c|c|c|}
\hline Synthetic Approaches & & & & icteristics Of Magneti & nanopart & & & & Ref \\
\hline nanoparticles & Synthesis & $\begin{array}{l}\text { Size and Size } \\
\text { distribution }\end{array}$ & Morphology & $\begin{array}{l}\text { Magnetization } \\
\text { values }\end{array}$ & Solvent & $\begin{array}{l}\text { Reaction } \\
\text { Temperature }\end{array}$ & Advantages & Disadvantages & \\
\hline $\begin{array}{l}\text { Bulk solution method } \\
\text { (Coprecipitation) }\end{array}$ & Simple & $10-50 \mathrm{~nm}$ & Spherical & $\begin{array}{l}20-50 \quad \mathrm{emu} / \mathrm{g} \\
\text { accompanied by } \\
\text { super- } \\
\text { paramagnetic } \\
\text { behavior }\end{array}$ & Water & $20-90{ }^{\circ} \mathrm{C}$ & Bulk synthesis & $\begin{array}{l}\text { Abandoned } \\
\text { oxidation } \\
\text { of magnetite to } \\
\text { maghemite, } \\
\text { diamagnetic } \\
\text { contribution }\end{array}$ & $45,47,48$ \\
\hline
\end{tabular}




\begin{tabular}{|c|c|c|c|c|c|c|c|c|c|}
\hline $\begin{array}{l}\text { Micro } \\
\text { emulsion method }\end{array}$ & Complex & $4-15 \mathrm{~nm}$ & $\begin{array}{ll}\text { Cubic or } \\
\text { Spherical }\end{array}$ & $>30 \mathrm{emu} / \mathrm{g}$ & Organic & $20-50{ }^{\circ} \mathrm{C}$ & $\begin{array}{l}\text { Uniform } \\
\text { properties }\end{array}$ & $\begin{array}{l}\text { Surfactants are } \\
\text { hard to } \\
\text { eliminate, } \\
\text { insignificant } \\
\text { quantity can be } \\
\text { produced }\end{array}$ & $45,49-51$ \\
\hline $\begin{array}{l}\text { Pyrolysis } \\
\text { (Aerosol/vapor) method }\end{array}$ & Simple & $\begin{array}{l}\text { 5-60 } \mathrm{nm} \text { and } \\
\text { wide size } \\
\text { distribution }\end{array}$ & Spherical & $\begin{array}{l}10-50 \mathrm{emu} / \mathrm{g} \text { in } \\
\text { addition to } \\
\text { preferred magnetic } \\
\text { characteristics }\end{array}$ & Organic & $5-60{ }^{\circ} \mathrm{C}$ & $\begin{array}{l}\text { High } \\
\text { production rate }\end{array}$ & $\begin{array}{l}\text { Large } \\
\text { aggregates are } \\
\text { formed }\end{array}$ & 45,52 \\
\hline Sol-gel method & Simple & $20-200 \mathrm{~nm}$ & Spherical & $\begin{array}{l}10-40 \quad \mathrm{emu} / \mathrm{g} \\
\text { alongside } \\
\text { paramagnetic } \\
\text { property }\end{array}$ & Water & $100-200{ }^{\circ} \mathrm{C}$ & $\begin{array}{l}\text { Desired shape, } \\
\text { length } \\
\text { and hybrid } \\
\text { nanoparticles }\end{array}$ & $\begin{array}{l}\text { Products } \\
\text { contain sol-gel } \\
\text { matrix } \\
\text { components }\end{array}$ & $45,53,54$ \\
\hline Gas deposition method & Complex & $\begin{array}{l}5-50 \mathrm{~nm} \text { and } \\
\text { narrow size } \\
\text { distribution }\end{array}$ & Spherical & $>20 \mathrm{emu} / \mathrm{g}$ & Organic & $900-1100{ }^{\circ} \mathrm{C}$ & $\begin{array}{ll}\text { Beneficial for } \\
\text { thin film } \\
\text { deposition and } \\
\text { protective }\end{array}$ & $\begin{array}{l}\text { Requires very } \\
\text { high } \\
\text { temperature }\end{array}$ & 45,55 \\
\hline
\end{tabular}

Due to extensive applications of MNPs, in the field of medicine, material science, engineering, and environmental sciences, different methods have attracted much attention. A lot of work has been devoted to synthesize shape-controlled, stable, biocompatible nanoparticles of uniform size. Monodispersity in nanoparticles with the tunable size is essential for biological and biomedical applications. Different methods have been developed to synthesize MNPs with sizes in the range of nanometers. The main challenge in the methods of preparation of MNPs for theranostic applications is to control the size, shape, distribution, magnetic properties, and surface chemistry of the nanoparticles. ${ }^{56}$ For in vivo applications, either biological or chemical methods are used to prepare magnetically sensitive nanoparticles. However physical approaches have also been employed for the fabrication of nanoparticles used for in vitro applications such as catalytic activities and metallurgical processes and many other industrial operations. The design and application of magnetic material propose some attractive potentials for biomedical application on account of their controllable sizes going from one to tens of nanometres, equivalent to the size of the cell $(10-100 \mu \mathrm{m})$, a protein $(5-50 \mathrm{~nm})$ a virus $(20-450 \mathrm{~nm})$, or a gene ( $2 \mathrm{~nm}$ wide and $10-100 \mathrm{~nm}$ long). ${ }^{57}$ 
Once these ferromagnetic nanomaterials are obtained, it is important to characterize these particles to determine their physical properties like shape, surface morphology, and size, thermal and magnetic characteristics. Several techniques have been designed to characterize nanoparticles, although none of these can provide complete information regarding studied materials. $^{58,59}$ Over with careful investigation of characteristics of materials, significant deductions of fundamental connections between them and applications can be made.

\section{SURFACE FUNCTIONALIZATION AND MODIFICATION OF MAGNETIC NANOPARTICLES}

Functionalized IONPs are potential candidates for biomedical applications because they possess exclusive physical and chemical properties. Magnetic nanoparticles need to have appropriate monodispersity, core size, high saturation magnetization (Ms), and acceptable hydrodynamic diameter for theranostic applications. ${ }^{60}$ They should have good constancy in biological fluid media, biodegradability and biocompatibility with decreased toxicity, and capability of clearance from the body. Therefore, it is essential to acquire water-dispersible nanoparticles. Mostly biological aqueous solutions are neutral, therefore coating of magnetic particles with polymers and surfactants to prevent agglomeration, increase biocompatibility, and supplement surface functionality. These interactions of coatings and magnetic core make a chaotic magnetic deposit, decreasing the overall magnetic phase, which is a vital factor for countless purposes. ${ }^{61}$ Coating is vital for colloidal stability of particles that prevents gravitational settlement and aggregation with decreasing the dipole-dipole interaction among particles. ${ }^{62}$ Surface modification of nanoparticles with peptides, antibodies, ligands, genes, drugs to make hybrid multifunctional nanomaterials, shown in Figure 2, not only confirms of stability of colloids but also prevents agglomeration, sedimentation, and oxidation. ${ }^{63,64}$ Some methods to improve colloidal stability include:

(a) Nanoparticles coating with organic species (polymers and surfactants).

(b) Nanoparticles coating with an inorganic species (silica or carbon). 


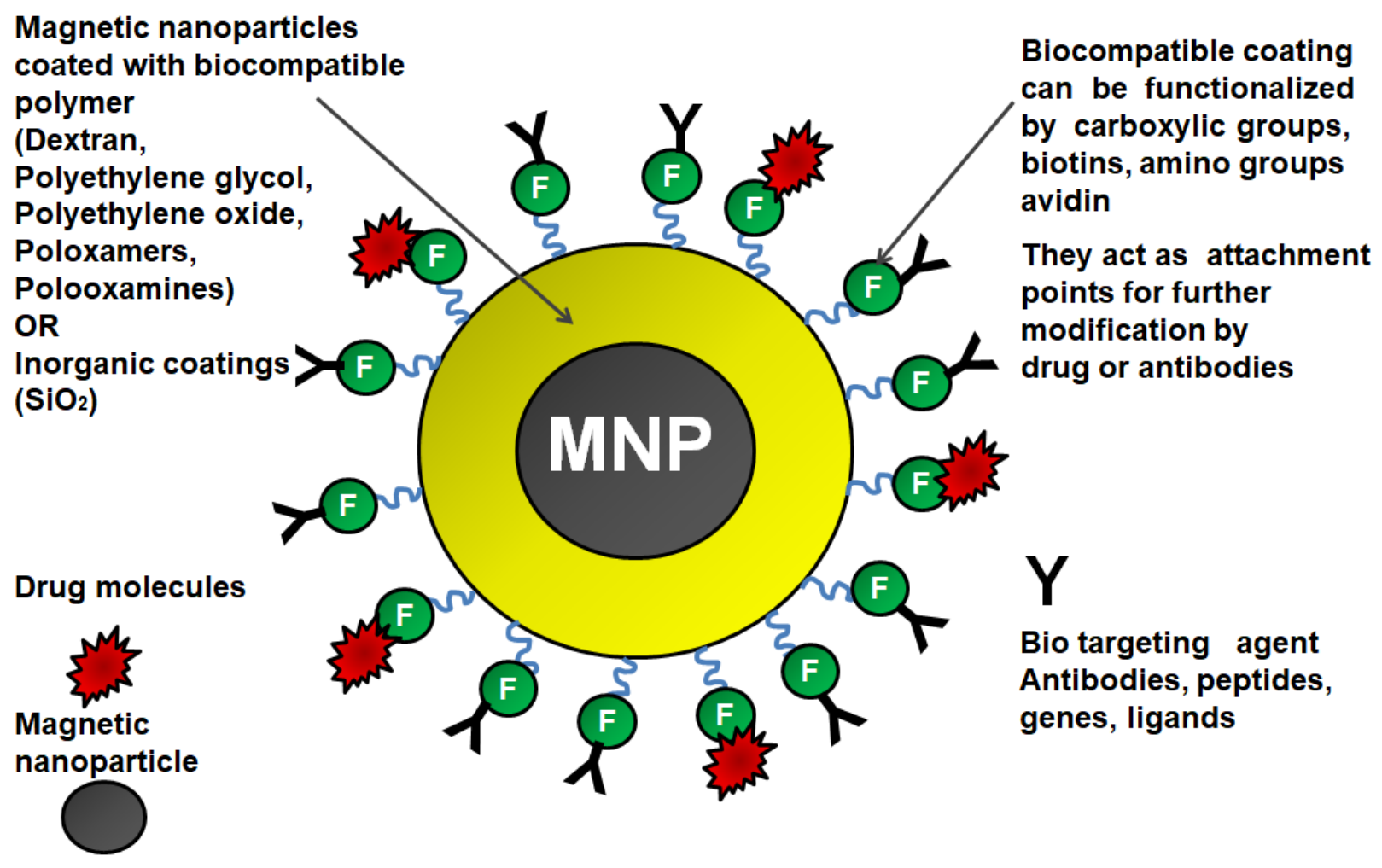

Figure 2. Surface stabilization of magnetic nanoparticles by peptides, antibodies, ligands, genes, and drugs, for the fabrication of multifunctional magnetic nanoparticles for theranostic applications.

Magnetic nanoparticle stability is very significant for theranostic applications. Quite a lot of methods were established for modification of IONPs comprising post-synthesis coating and in situ coatings, which are trivial ways for coating of IONPs with organic material. MNPs are coated with a diverse group of organic and inorganic materials to prevent particle accumulation. ${ }^{65}$ They act as stabilizers by binding covalently to the nanoparticles that enable the nanoparticles to possess high magnetic susceptibility. Magnetic nanoparticle coating can be achieved via several methods, counting in situ coatings, post-synthesis end grafting, and adsorption. ${ }^{66} \mathrm{An}$ additional main feature of surface functionalization to transmit stability involves the combination of additional stimuli-responsive functions with molecules. Responsive magnetic colloids have functional groups that respond to the environs in a well-controlled way. That is why the surface chemistry of magnetic colloids is notably reliant on the kind of functional groups. IONPs were modified with exceptional groups (e.g. $-\mathrm{OH},-\mathrm{NH} 2,-\mathrm{COOH},-\mathrm{SH}$ ) for added functionalization by attachment of diverse biologically active molecules, resulting in organic/inorganic complex having core/shell nanostructure for different applications. ${ }^{67,68}$ Recognition time or treatment of 
unhealthy cells through immune system is prolonged by adding functionality. These functional complexes possess theranostic properties and can be employed in both therapeutic and diagnostic procedures. The surface chemistry of these core-shell nanostructures dictates their working environments and function. ${ }^{69}$ The structure of magnetic polymer colloids not only preserved the magnetic property of magnetic iron oxides but also other properties of organic molecules. The surface modification brings a precise variation in the behavior of IONPs in solution such as overall toxicity and $\mathrm{pH}$ response. ${ }^{70,71}$ By tuning the size of the core, the thickness of the coating, and target ligands, the nanoprobes were designed for targeting particular cells, tissues, and various disease biomarkers. The core/shell morphology of MNPs has the benefit of high stability against oxidation, good dispersibility, and a large surface area to load drugs onto the shell. ${ }^{72}$ Scientifically, spherical nano architectures displaying blends of some characteristics are particularly attractive because discrete material does not own such properties. Biodegradable polymer colloids are small particles having diameter extending from 10- $400 \mathrm{~nm}$ and are utilized for different applications since 1970s. Properties of these nanostructures get vary with morphology, formulation parameters, constituents, and design. The use of recent investigation methods enhances knowledge of the superficial and internal morphology of the hybrid nanoparticles and offers a better understanding of the colloidal behaviour. MNPs formulation with polymers offers a multifunctional colloidal system comprising both imaging and therapeutic agents. Because of exceptional properties, magnetite has been evaluated as a promising material for diagnostic, therapeutic, or simultaneous diagnostic and theranostic purposes. ${ }^{73}$ Several techniques have been implemented for enclosing the magnetite nanoparticles and other therapeutic agents (drug) inside polymer shells including emulsification, single or double solvent evaporation technique, spray drying, or phase separation. The formulation method determines the type, size, entrapment efficiency, and release profile of a drug.

To improve the physical properties by surface modification of IONPs, different processes for encapsulation were introduced which include chemical and physical methods by using polymers, organic surfactants, inorganic compounds, and bioactive molecules. ${ }^{74}$ Appropriate surface modification can also import the MNPs with better biocompatibility, long blood circulation and further functionalization, such as active targeting ability. ${ }^{75}$ The chemical method depends on the heterogeneous polymerization of chemically or inherently altered inorganic nanoparticles being encapsulated in the dispersion medium. Different forms of polymerization 
like miniemulsion, microemulsion, and emulsion were used in dispersion media. Emulsion polymerization has been explored and reported for iron oxide encapsulation in polystyrene particles to acquire magnetic latex particles for biomedical applications. ${ }^{76}$ But these particles have non-homogeneous encapsulation and poor encapsulation productivity that makes it difficult or even in some cases impossible to produce complex structures. Therefore this approach is often constrained. In the process of miniemulsion polymerization, two phases (surfactants or monomers comprising aqueous phase) are transformed into colloidal particles stabilized by surfactant. ${ }^{77}$ The main benefit of the mini-emulsion technique is the effective control of the number of final particles by regulating the number of initial droplets and ultimately the size of final particles. There is no diffusion of monomer(s) in this process which imparts exceptional characteristics to inorganic nanoparticles, as widely stated in the literature. ${ }^{78}$ By using the technique of miniemulsion polymerization, it has been widely stated that the magnetic latexes can be obtained by encapsulating IONPs through polystyrene particles that show various applications in industry and mainly in the biomedical sector. ${ }^{76}$ Microemulsion polymerization is the most widely spread technique to prepare well-defined stable nanoparticles of precise shape and size ${ }^{79}$ Since the early 1980 s, water in oil (w/o) or inverse microemulsion mechanism has been utilized to prepare metal nanoparticles as well as minerals. The technique of microemulsion has various advantages in dispersion medium over all other polymerization techniques and offers the possibility to prepare a wide range of colloidal particles with diverse characteristics. ${ }^{80}$ This approach is not successful at the industrial level owing to large oil (solvent) quantity usage, surfactants, and poor nanoparticle yield. ${ }^{80}$ During the polymerization process, the low enclosed quantity of IONPs is due to the inorganic nanoparticles, being desorbed. This approach leads to a self-assembly method that was created and mainly used to enclose nanoparticles on seed colloidal particles through simultaneous adsorption of oppositely charged polyelectrolytes and inorganic nanoparticles. ${ }^{81}$ Adsorption of polymer is through attractive electrostatic interaction, by thiol groups, or via specific interactions (for example pairing of ion, dipole-dipole, complexation, etc.). ${ }^{82}$ Some nanoparticles encapsulation in a polymer microsphere results in an unavoidable phenomenon of aggregation. Among all, a double emulsion technique is the most fascinating method that depends on the emulsification of aqueous phase into oily phase (w/o) or in reverse $(\mathrm{o} / \mathrm{w})$. It was first developed for hydrophobic and hydrophilic active molecules encapsulation. ${ }^{83}$ To acquire w/o/w emulsion, first of all, water was emulsified in oil and then 
converted to the water phase. The hydrophobic MNPs encapsulation involves the full dispersion of polymer in the organic process. The process and formulation parameters principally regulate the size distribution, morphology, size, and zeta potential of these colloidal hybrid dispersions that result in hybrid submicron particles. ${ }^{83,84}$ All the factors concerning the properties of colloidal hybrid particles were studied by encapsulating IONPs for in vivo bioimaging and therapeutic applications (therapy and test tube treatment simultaneously). ${ }^{85,86}$ The double emulsion method is considered as the most popular and appropriate technique to control the process parameters for the synthesis of colloidal or composite stable systems consisting of nanoparticles of small size and narrow distribution. ${ }^{87}$ In a double emulsion process of water-oilwater (w/o/w), two aqueous phases are isolated by the oil phase.

\section{THERANOSTIC APPLICATIONS OF MAGNETIC NANOPARTICLES}

Magnetic nanoparticles carrying organic or inorganic surface coatings have attained increasing consideration in the field of material sciences because the subsequent tools permit controllable modalities for progressive biomedical applications from diagnostic and therapeutic to bioseparations. Because of their stability and functionality, they have been principally exploited to boost contrast and therapeutic performance of MRI, ${ }^{88-90}$ for targeted drug delivery and application of hyperthermia ${ }^{91-93}$ together with precise capture of selected nucleic acid with high

accuracy. ${ }^{94}$ Molecular imaging is vital for characterizing biological methods to efficiently exploit the magnetic colloids for diagnosis at the cellular and subcellular levels. The presence of magnetic colloids in the body creates an additional field that increases the relaxation rate by inducing local field inhomogeneities leading to contrast in the image. IONPs have been bound with antibodies, peptides, proteins, and small molecules and proteins and are used for diagnosis and therapy. ${ }^{3}$

Multi stimuli-responsive platforms, consisting of composite/hybrid core-shell systems, resulted by incorporation of MNPs with polymers are used for both diagnosis and treatment. ${ }^{95}$ The core-shell structure combines the magnetic behavior of MNPs with the flexibility of an organic coating that imparts new and specific properties such as stability in chemical and physical properties, ecologically safe, biocompatibility. The capability of being regulated through an external magnetic field offer convenient ways of labelling of biomolecules magnetically following very efficient bio-separation, acute biosensing, imaging, contrast 
improvement, hyperthermia, and also drug delivery to their specific sites bioproducts. ${ }^{96} \mathrm{~A}$ brief overview of MNPs used in "conventional" biomedical applications associated to theranostics is given below:

Magnetic Nanoparticles in Magnetic Resonance Imaging (MRI). New imaging technologies have been evolved due to development in nanotechnology, cellular and molecular biology, and imaging modalities. ${ }^{97}$ Biomedical imaging via MRI is used to image contrast formed as a tissue response to the outside magnetic field that is directed by the proton density of proton and magnetic relaxation time. Researchers explored the usage of MNPs to increase the contrast of signal and advance the soft tissue image resolution as presented in Figure 3 for in vitro $\mathrm{MRI}$ and in vivo MRI investigations.

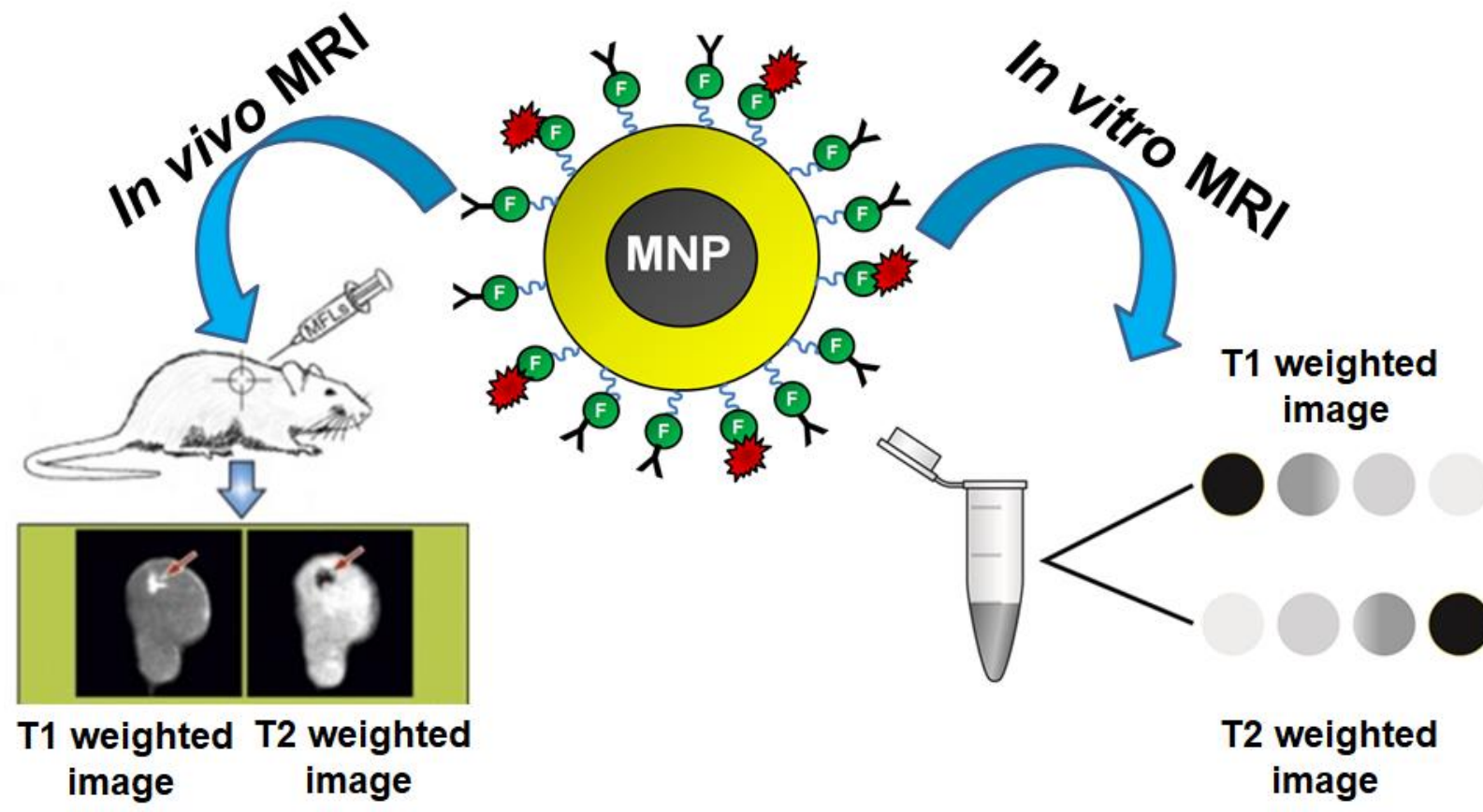

Figure 3. T1 and T2 signal enhancement by magnetic nanoparticle as a contrast agent used in in vivo MRI and in vitro MRI. Reprinted from ref ${ }^{98}$. Copyright 2021 Elsevier.

At present, contrast agents have been investigated in over $30 \%$ of MRI studies. In clinical applications, the high spatial MRI resolution and MNPs precision to target permits the tumor imaging of size 2-3 mm. ${ }^{99}$ SIONPs conjugated with monoclonal antibody C95 (SPIONs-C595) having a hydrodynamic size of $87 \mathrm{~nm}$ were demonstrated as MRI imaging nanoprobes. They showed a significant reduction in $\mathrm{T}_{2}$-weighted signal intensities. SPIONs-C595 nanoprobe has 
the potential ability in $\mathrm{T}_{2}$-weighted $\mathrm{MRI}$ contrast agents towards recognizing breast cancer (MCF-7) cells. ${ }^{100}$ The novel magnetic composite nanoparticle c(RGDyK)-PDA-SPIONs having $50 \mathrm{~nm}$ size exhibited a substantial signal decrease with the increasing nanoparticle concentration. These composites were not toxic at lesser concentrations ( 5 to $20 \mu \mathrm{g} / \mathrm{mL}$ ) and have a noteworthy targeting outcome on liver cancer cells. Experimental data of animals proved that prepared composites are potentially good T2 contrast agents that enhanced imaging of HepG2 human hepatocellular carcinoma present in mice. ${ }^{99}$ Tailored-made MNPs with a $15 \mathrm{~nm}$ core size layered by polyethyleneglycol (PEG) were uptaken by both macrophages and tumoral cells without any toxicity in in vitro in addition to in vivo experiments. ${ }^{101}$ Prepared nanoparticles were effusively taken by the tumor after injection presenting extraordinary potential as MRI real-time contrast agents and for continuous monitoring of the development of a tumor. For the identification of tumors in the body tissues, SPIONs were developed and tried for in vivo MRI as a contrast agent. In one of the major study, extremely diluted magnetic emulsion $(0.05 \mathrm{wt} \%)$ presented substantial T2 contrast improvement in MRI images at the concentration of $500 \mu \mathrm{L}$, representing the dispersal of magnetic colloids within body tissues, for instance, the spleen, liver, and kidneys of rats (Sprague dawley). It is helpful for advanced examinations particularly in theranostic applications of an emulsion comprising of magnetic nanoparticles as MRI contrast agents. ${ }^{102}$ Recently some researchers also developed iron-based T1 MRI contrast agents showing a positive contrast effect. The non-toxic and biocompatible melanin-like PEGylated nanoparticles conjugated with ions of paramagnetic $\mathrm{Fe}^{+3}$ ((PEGylated $\left.\mathrm{Fe}^{+3}-\mathrm{MelNPs}\right)$,) displayed a bright signal with improvement after intravenous injection in spleen and liver of healthy mice. ${ }^{103}$ Silica-coated SPIONs $\left(\mathrm{Fe}_{3} \mathrm{O}_{4} @ \mathrm{SiO}_{2}\right)$ of average size of 30-40 nm exhibited in vivo $\mathrm{T} 1$ contrast in the liver, heart, bladder, and kidney with unusual ability to increase MR images of healthy mice. ${ }^{104}$ An important role was played by surface ligands in enhancing the performance of MNPs in MRI applications. A distinctive natural macromolecule (protein-bovine serum albumin, BSA) and a synthetic macromolecule (poly (acrylic acid)-poly (methacrylic acid), PMAA-PTTM) were used for functionalization of magnetite nanoparticles to prepare nanoparticles of $\mathrm{Fe}_{3} \mathrm{O}_{4}$ - $\mathrm{BSA}$ and $\mathrm{Fe}_{3} \mathrm{O}_{4}$-PMAA-PTTM having comparable dimension and magnetization utilizing coprecipitation method. They were investigated and equated in in vitro and in vivo MRI performances. $\mathrm{Fe}_{3} \mathrm{O}_{4}$ BSA showed a greater $r_{2} / r_{1}$ ratio within the solution and dark signal for kidney and liver target sites. Whereas $\mathrm{Fe}_{3} \mathrm{O}_{4}$-PMAA-PTTM presented a very low $r_{2} / r_{1}$ ratio within a solution and a 
positive contrast in kidney and liver sites under $\mathrm{T}_{1}$-weight imaging in mice. ${ }^{105}$ The thickness of the organic coating also affects the relaxometric properties of nanoparticles. Different types of IONPs with various coating layer thickness, prepared via microwave-driven synthesis method. IONPs having a thin layer of coating generated an excellent positive T1 MRI contrast in both brain and body MRI images of healthy mice. ${ }^{106}$ An in vivo MRI of the heart chamber and vena cava of healthy mice by very small PEGylated IONPs (VSIONPs) having $3.5 \mathrm{~nm}$ sizes showed positive contrast. ${ }^{107}$ Magnetic latex particles were productively prepared through adsorption of aminodextran (AMD) polymer over oxidized dextran; AMD was synthesized using chemical grafting of hexamethylenediamine. The prepared magnetic nanoparticles demonstrated good capability for in vivo diagnostic applications and confirmed greater T2 contrast-ability in comparison with gadolinium in MRI. ${ }^{108,109}$

Researchers worldwide have also been working persistently for developing multimodal T1- and T2 contrast agents, accompanied by high sensitivity along with an accurate resolution of MRI imaging based on MNPs. Nitrodopamine-PEG grafted single-core truncated cubic IONPs (NDPEG-tNCIOs) were developed which were able to generate noticeable dual MRI contrasts in MRI with better transverse and longitudinal relaxivities of $791 \pm 38.39$ and $32 \pm 1.29 \mathrm{mM}^{-1} \mathrm{~s}^{-1}$, respectively. ${ }^{110}$ Manganese-doped iron oxide nanocomposites (Mn-IONPs@PEG) were ultimate candidates as dual $\mathrm{T}_{1} / \mathrm{T}_{2}$ contrast agents showing exceptional $r_{1}$ and $r_{2}$ in vitro and in vivo experiments. $^{111}$

Recently MNPs have been combined with other nanoparticles for multimodal imaging methods to enhance the imaging signal for the early detection and treatment of brain pathologies. MNPs are so diverse and can be used for bimodal imaging. MNPs have been explored in computed tomography (CT) as multimodal contrast agents in combination with other nanoparticles such as gold, to diagnose diseases or internal injuries in blood vessels, bones, soft tissues, and other parts of the body. The hybrid nanoparticles have the potential to be used as CT contrast agent to enhance the contrast of biological and non-biological materials/samples because of high X-ray attenuation and high density. Decoration of MNPs with functional groups not only reduces their toxicity but also give them targeting properties where special organ and/or tissue can be targeted. ${ }^{112}$ A novel multimodal consisting of superparamagnetic gold-coated iron oxide nanoparticles have been examined as contrast agents in phantoms for X-ray CT and T2-weighted MRI. The gold coating could enhance the contrast of these nanoparticles when irradiated with X-rays and 
magnetic content is a well-known source of negative contrast in MRI. As expected, for the X-ray CT phantoms, there is a linear increase of contrast with concentration and T2-weighted MRI images become substantially darker as the concentration of iron increases. The obtained results and their comparative analysis show that these kinds of hybrid nanoparticles can effectively be used as contrast agents in MRI and CT and could be an interesting starting point for future applications as multimodal contrast agents for targeted imaging. ${ }^{113}$ The novel gold-iron oxide janus magnetic-plasmonic nanoparticles were prepared as versatile nanoprobes for multimodal imaging. The nanoparticles were characterized as contrast agents for different imaging techniques, including X-ray computed tomography (CT), T2-weighted nuclear magnetic resonance imaging (MRI). Particle size and morphology effects the performance of these developed nanoparticles as contrast agents and showed the advantage of a Janus configuration. The results obtained with these non-invasive techniques show the high versatility of these nanoparticles, the advantages of a Janus configuration, and their high potential in multipurpose biomedical applications. ${ }^{114}$

Hydrophilic and carboxyl-functionalized $\mathrm{Fe}_{3} \mathrm{O}_{4}$ nanoparticles and amino-functionalized gold nanospheres (Au- SH-PEG-NH ) were separately synthesized. The Au nanoparticles were conjugated with hydrophilic $\mathrm{Fe}_{3} \mathrm{O}_{4}$ nanoparticles through an amide reaction to obtain $\mathrm{Au}$ $\mathrm{Fe}_{3} \mathrm{O}_{4}$ nanohybrid composites. They show high $r_{2}$ relativity $\left(157.92 \mathrm{mM}^{-1} \mathrm{~s}^{-1}\right)$ and a Hounsfield units (HU) value $(270 \mathrm{HU})$ at $\mathrm{Au}$ concentration of $8 \mathrm{mg} / \mathrm{mL}$ and could be practical as nanoprobes for the dual-modal MR/CT imaging of a xenografted tumor model. This facile method can be extended to prepare other multifunctional nanoparticles for dual-modal MR/CT imaging having excellent T2 and CT performance. ${ }^{115}$ The synthesis of composite bismuth- dextran-coated iron oxide nanoparticles (BION) are clinically approved as contrast agents for both CT and MRI. BION CT phantom images revealed that the X-ray attenuation of the different formulations was dependent upon the amount of bismuth present in the nanoparticle, while T2-weighted MRI contrast decreased with increasing bismuth content. No cytotoxicity was observed in Hep G2 and BJ5ta cells in in vivo experiments performed in mice using a micro-CT and a 9.4 T MRI system. Thus, dextran coated BION are biocompatible, biodegradable, possess strong X-ray attenuation properties and also can be used as T2-weighted MR contrast agents. ${ }^{116}$ MNPs have been also utilized in ultrasonic CT (UCT) imaging performed on a breast mimicking phantom and on an ex vivo tissue model. It showed an improvement in contrast to noise ratio and can also serve as a 
pre-screening platform for disease diagnosis. Moreover, it was suggested that these MNPs can be applied for multimodal MRI-CT imaging purposes that allowed the detection of the particles existence and location, in both raster-scan projection and UCT imaging. ${ }^{117}$ The theranostic agent $\left(\mathrm{Au} / \mathrm{PPY} @ \mathrm{Fe}_{3} \mathrm{O}_{4}\right)$ based on the cointegration of gold nanorods (Au NRs) and superparamagnetic iron oxide $\left(\mathrm{Fe}_{3} \mathrm{O}_{4}\right)$ into polypyrrole was developed. They produce high contrast for magnetic resonance (MR) and X-ray computed tomography (CT) imaging. Cell viability assay showed that these nanocomposites had low cytotoxicity and the cancer cells can be efficiently killed by the photothermal effects of the $\mathrm{Au} / \mathrm{PPY} @ \mathrm{Fe}_{3} \mathrm{O}_{4}$ nanocomposites. These nanocomposites demonstrate highly versatile multi-functionality with a great potential in simultaneous multimodal imaging-guided cancer theranostic applications. ${ }^{118}$ These bimodal agents provided dual/multipurpose contrast enhancement and have strong potential to be able to take place in the market. However, further research was needed to shed light on the clinical potential of metallic nanoparticles.

Magnetic Nanoparticles for Hyperthermia Treatment. Hyperthermia has been extensively explored using biocompatible MNPs as heat mediator for cancer therapy because of high efficacy and with some degree of side effects. It became an innovative and new heat therapy within which the heat-producing agents are MNPs. Magnetic treatment modality contributes to presently existing methods for cancer treatments like gene therapy, chemotherapy, surgery, immunotherapy, and radiation therapy. The heat generated by MNPs in a non-intrusive applied alternating magnetic field (AMF) to tumor cells, contributes to cell death via irretrievable physiological changes known as magnetic hyperthermia displayed in Figure 4. ${ }^{119}$ 


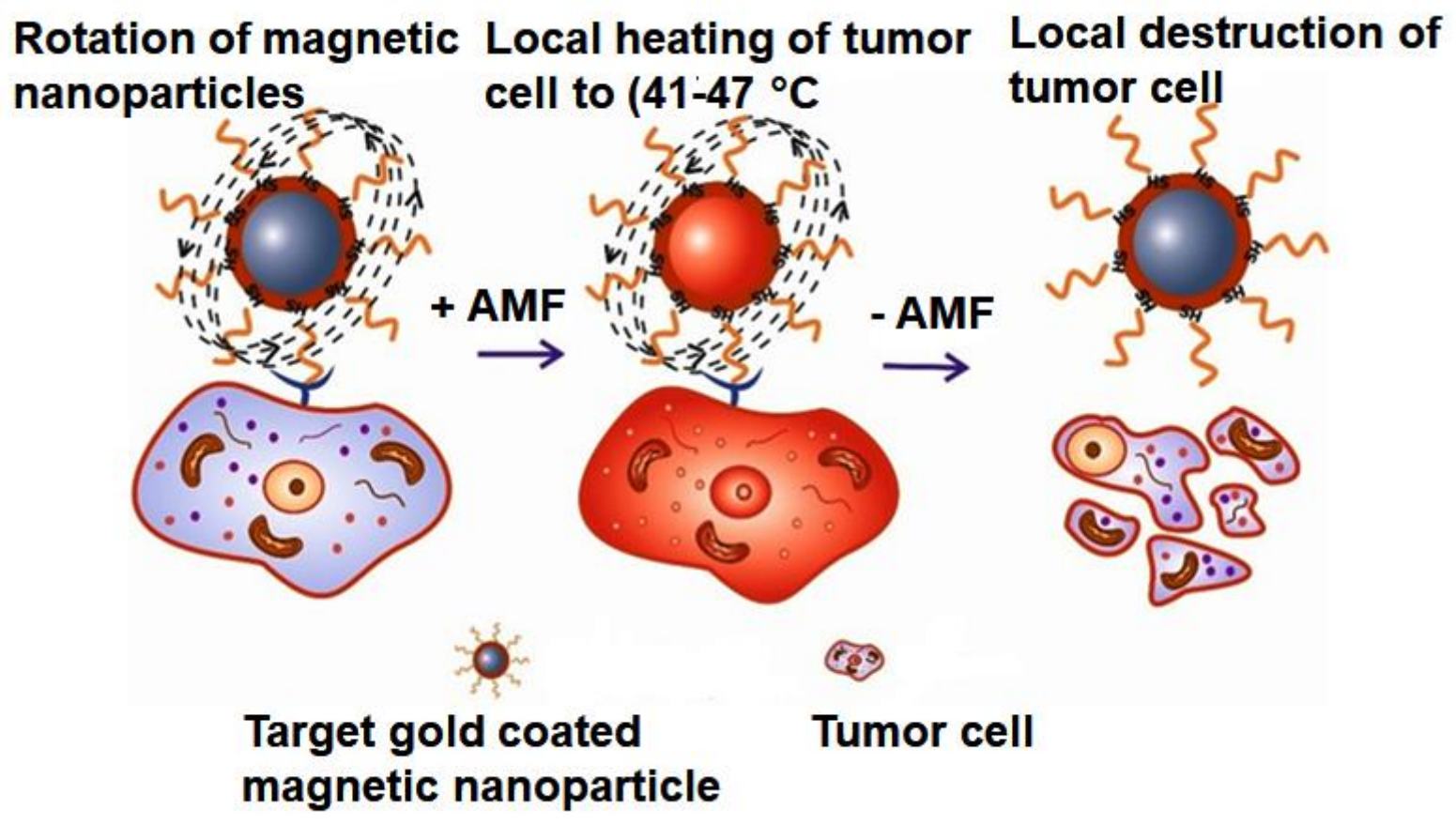

Figure 4. Magnetic hyperthermia principle. Magnetic nanoparticles are targeted towards cancer cells in an alternating magnetic field (AMF). Afterward, the heat generated by the magnetic nanoparticles, causes confined heating of cancer cells in the middle of 41 to $47^{\circ} \mathrm{C}$, Reprinted from ref ${ }^{120}$. Copyright 2020 Multidisciplinary Digital Publishing Institute.

The MNPs could be cast to the target area and heated up by an externally applied alternating current magnetic field. As stated by Pankhurst, the rate of heat deposition is $100 \mathrm{~mW} / \mathrm{cm}^{3}$ which is usually needed, and the field frequency should be within the $\mathrm{kHz}$ range with an amplitude of a few $\mathrm{kA} / \mathrm{m} .{ }^{89}$ Generally, iron oxides have their applications for hyperthermia treatment. Further, MNPs that have stretched substantial clinical recognition are IONPs coated with either dextran or liposomes. Wang et al. originated chitosan MNPs as T2 contrast agent encapsulating doxorubicin and also plasmid DNA and focused on lung tissues prone to cancer. ${ }^{121}$ The composites showed sharp T2 weighting contrast enrichment combined with enhanced tumors imaging with commendable therapeutics delivery and capability for recording real-time therapeutics. Many new IONP-derived products were established, some of them were approved by the FDA and the European Commission (EC) for clinical use (Table 1). Moreover, the growing number of clinical trials consuming these IONPs extended their potential hyperthermia applications. ${ }^{14}$

Hybrid nano-magnetic structures also showed higher SAR values as compared to bare 
nanoparticles due to greater stability in aqueous solutions. Heat is delivered to tumor cells by them without any harmful undesirable effect to surrounding cells in vitro and in vivo. ${ }^{122}$

Silica coated biocompatible IONPs were incorporated with antibodies (anti- $\alpha v \beta 6$ ) to target $\alpha v \beta 6$, an oral squamous cell carcinoma biomarker. The prepared antibody-targeting MNPs in an externally applied alternating magnetic field caused thermal ablation of tumors. It offered a favorable therapy for the targeted treatment of oral squamous cell carcinoma. ${ }^{123}$ IONPs (15-35 $\mathrm{nm}$ ) coated with silica induces magnetic hyperthermia in tumor cells (L929 and HeLa cells) and effectively killed them in vitro under induction heating. ${ }^{124}$ Hydroxyapatite coated iron oxide (IOHAp) nanoparticles showed a very high heating efficiency as nano heaters with $85 \mathrm{~W} / \mathrm{g}$ SAR value at 180 Gauss magnetic field strength with $409 \mathrm{kHz}$ frequency. All of the MG-63 osteosarcoma cells under experimentation were killed in nearly $30 \mathrm{~min}$ exposure. The cellular system was denatured and oxidized due to damage in nucleotides, proteins, and membranes by internalization of these nanoparticles leads ultimately cell death. ${ }^{125}$ In vivo and in vitro hyperthermia experimentation on nanofluids $\left(\mathrm{Mg} 0.13-\gamma-\mathrm{Fe}_{2} \mathrm{O}_{3}\right)$ was carried out for evaluation of their heating capability. $\mathrm{Mg} 0.13-\gamma-\mathrm{Fe}_{2} \mathrm{O}_{3}$ nanospheres $(22.8 \mathrm{~nm})$ showed significant heat induction characteristics due to the dramatically enhanced magnetic susceptibility. It resulted from the systematic distribution of $\mathrm{Mg}^{2+}$ cations in octahedral site of $\gamma-\mathrm{Fe}_{2} \mathrm{O}_{3}{ }^{126}$ The in vitro hyperthermia investigations of the polycaprolactone (PCL)-coated SPIONs on human liver cancer cells (HepG2) cell viability in alternating magnetic field presented an innovative therapeutic agent. These nano heating agents exhibit lower systemic toxicity, thermosensitivity, and improved cytocompatibility in externally applied alternating magnetic field of different frequencies. ${ }^{127}$ Heating efficiency of magnetic colloidal nanoparticles emulsified by sodium dodecyl sulphate (SDS) aqueous solution was also evaluated, showing significant potential for therapeutic applications. ${ }^{128}$ Water-soluble magnetite $\mathrm{Fe}_{3} \mathrm{O}_{4}$ nanoparticles coated by Eudragit E100 (amino methacrylate copolymer) encapsulating Doxorubicin drug were explored for hyperthermia. The specific absorption rate values were also calculated and estimated to be 2.41 , 2.71 , and $4.28 \mathrm{~W} / \mathrm{g}$ at the frequency of 259,327 , and $518 \mathrm{kHz}$ and magnetic field strength of 22 , 17, and $23 \mathrm{mT}$, respectively. The well-controlled SAR values of novel colloids specified their forthcoming usage for hyperthermia. ${ }^{87}$

Reactive Oxygen Species (ROS) play important vital roles in many cellular signaling paths under both physiological and pathological conditions. Few evidences also showed an effect of 
magnetic field on ROS levels depending upon its intensity/frequency/exposure time. ${ }^{129}$ External environment of MNPs alone or combined with magnetic field could influence ROS production in cells leading to more cell injury and death. ${ }^{130}$ Recent studies showed that the fundamental mechanisms of temperature rise are not fully pronounced yet. It is difficult to measure the macroscopic temperature rise in magnetic hyperthermia in cultures incubated with a low concentration of magnetic nanoparticles and in an alternating magnetic field of low intensity. Therefore, the invention of intracellular Reactive Oxygen Species (ROS) as a mechanism was investigated to induce cell death under these conditions. The ROS effect on the viability of HepG2 human hepatoma cells was in the presence of alternating magnetic fields using hyperthermia agents, such as biomimetic MNPs (BMNPs) intervened by magnetosome MamC protein from Magnetococcus marinus MC-1. The results demonstrated an increase in intracellular ROS production up to 90 in AMF leading to loss of cell viability (up to 40\% reduction) deprived of the rise in temperature. ${ }^{131}$

However, the improvement of effective and better heat nanomediators exhibiting an extraordinary specific absorption rate (SAR) value remains required for quantifiable clinical applications. They are essential to overcome the numerous restraints encountered before because of the high amount of nanomaterial mandatory aimed at operative treatment. The values of SAR are effected by the morphology and composition of the MNPs, also be effected by the property of magnetic field such as frequency (f) and amplitude $(\mathrm{H})$. Therefore, for accomplishing an effect of hyperthermia to tumors, the magnetically mediated nanoparticles must show the high SAR and low (f)/(H) in the low content. SAR values of SPIONs can be improved by increasing either (f) or $(\mathrm{H})$ (or both increases) during the measurements. ${ }^{15}$

Clinical hyperthermia of prostate cancer remains a challenging problem. Clinical studies of intratumorally delivered MNPs for the application of thermotherapy in humans were initiated in 2007 and have been successfully conducted for patients with glioblastoma and prostate cancers. IONPs fluid transperineally was injected into the prostate of ten patients who received six thermal therapies of 60 minutes duration at weekly intervals. There is a maximum temperature rise of $55^{\circ} \mathrm{C}$ in the prostate with a medium temperature of $40.7^{\circ} \mathrm{C}$ within the tumour and $40.2{ }^{\circ} \mathrm{C}$ in peritumoural zones. They confirmed the practicability of the methodology with no major late treatment-related illness. ${ }^{132}$ Later, they performed an altered clinical assay representing that interstitial heating using IONPs was possible and well accepted in patients with 
locally recurring prostate cancer, and the retention of nanoparticles in the prostate was highly resilient. ${ }^{133}$ The hyperthermia treatment can control the more aggressive component of the tumor and stop or delay the need for radiotherapy or surgery in patients with regular glioblastoma multiforme. In this trial, patients received four to 10 treatments, attaining a temperature of $44.6{ }^{\circ} \mathrm{C}$. It was observed that IONPs were tolerated well by all patients with minor or no side effects. ${ }^{134}$ Hyperthermia in patients with metastatic bone tumors has been carried out through initial surgical intervention followed by the implantation of a mixture of "bare" magnetite nanoparticles and calcium phosphate cement, which is a biocompatible bone substitute. $32 \%$ of injuries were reduced and obtainable visible bone formation, $64 \%$ presented no advanced injuries for more than three months and just $4 \%$ presented a poor response to the treatment, indicating well-being and efficiency. ${ }^{135}$ Following these clinical trials, MagForce conducted a randomized, controlled trial (DRKS00005476) to control the effectiveness and safety of hyperthermia in combination with radiotherapy vs. radiotherapy alone to treat glioblastoma multiforme. ${ }^{15}$ Despite promising results of magnetic hyperthermia therapies, it is still in its very early stages. Numerous limitations must be fixed through advancements in biological and technical techniques before this new therapy becomes part of the standard of care for some cancers. So far, preclinical trials (Table 4) frequently apply field strengths, frequencies, or concentration of MNPs that are away from what is clinically possible, and thus, advance research is necessary for the areas of design, delivery, and the heating of nanoparticles, to accomplish clinical translation in the future. ${ }^{15}$ Future preclinical investigations should be focused on designing MNPs that can target and heat tumors more efficiently and successfully. Additionally, various hyperthermia enhancers should be assessed in combination with magnetic hyperthermia, with the eventual objective of attaining clinical practicability.

Table 4. In vivo investigation of biologically targeted hyperthermia. ${ }^{15}$

\begin{tabular}{llllll}
\hline Target & $\begin{array}{l}\text { Field } \\
\text { strength } \\
(\mathrm{kA} / \mathrm{m})\end{array}$ & $\begin{array}{l}\text { Frequency } \\
(\mathrm{kHz})\end{array}$ & $\begin{array}{l}\text { Concentration } \\
\text { of MNPs } \\
\text { delivered }\end{array}$ & $\begin{array}{l}\text { Targeting } \\
\text { mechanism }\end{array}$ & Results \\
Squamous & 38 & 980 & $1700 \mathrm{mg} / \mathrm{kg}$ & $\begin{array}{l}\text { Enhanced } \\
\text { Permeability }\end{array}$ & $\begin{array}{l}\text { Durable } \\
\text { ablation of }\end{array}$ \\
Cell & & & & and Retention & tumors in $84 \%$
\end{tabular}




\begin{tabular}{|c|c|c|c|c|c|}
\hline & & & & (EPR) & $\begin{array}{l}\text { of hyperthermia } \\
\text { group }\end{array}$ \\
\hline Melanoma & 5 & 366 & $13.30 \mathrm{mg} / \mathrm{kg}$ & EPR + Porphyrins & $\begin{array}{l}\text { Tumor volume } \\
\text { was smaller in } \\
\text { the } \\
\text { hyperthermia } \\
\text { group }\end{array}$ \\
\hline $\begin{array}{l}\text { Breast } \\
\text { Cancer }\end{array}$ & $56-113$ & 153 & $150 \mathrm{mg} / \mathrm{kg}$ & $\begin{array}{l}\text { EPR +Antibody } \\
\text { targeting integral } \\
\text { membrane } \\
\text { glycoprotein }\end{array}$ & $\begin{array}{l}\text { Tumor size } \\
\text { increases } \\
\text { significantly } \\
\text { except for the } \\
\text { groups that } \\
\text { received the } \\
\text { lowest energy }\end{array}$ \\
\hline
\end{tabular}

Magnetic Nanoparticles in Drug Formulation for Drug Delivery. Magnetic drug delivery systems consisting of a therapy component bound to MNPs superficial or enclosed in composites of MNPs and polymer. The significance of targeted drug delivery is that it delivers drugs directly to the center of disease in an external magnetic field, under varying constraints like an electric current, magnetic fields, temperature, light, and ultrasound without affecting the body shown in Figure 5. The therapeutic beneficial properties of magnetic nanoparticles are attractive and also interesting, counting their surface charge, charge density, chemical conformation, shape, inner morphology, size, degradation, and sensitivity to stimuli for the treatment of diseases. ${ }^{68,136}$ 


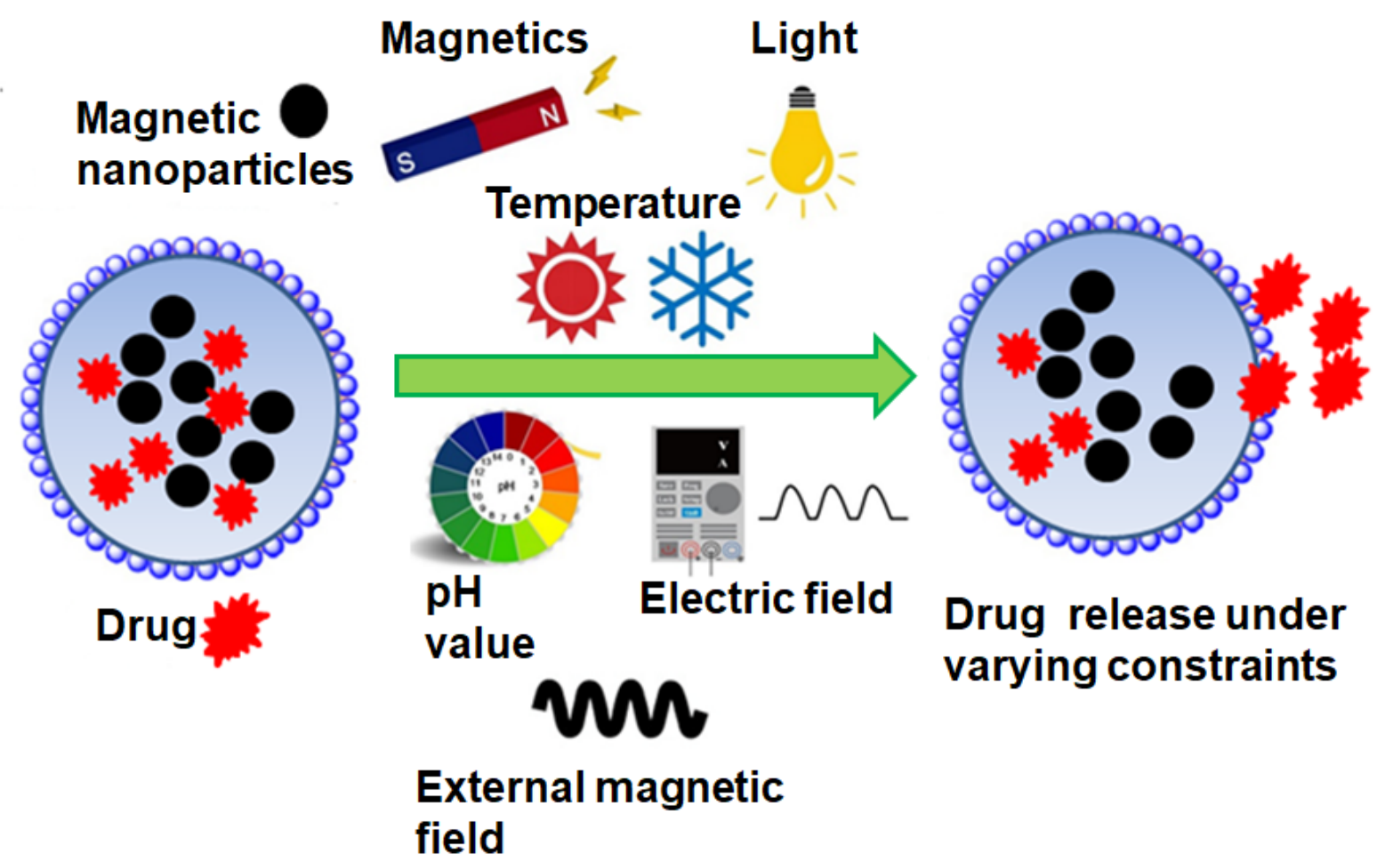

Figure 5. An illustration of the release of drug by a nanocomposite in an external magnetic field under varying constraints (pH, light, temperature, electricity, and magnetics).

Chertok et al. effectively transported polyethyleneimine (PEI)- magnetic nanoparticles (GPEI) to having a saturation of $93 \mathrm{emu} / \mathrm{g}$ to cancer cells of the brain with low cellular toxicity. ${ }^{137} \mathrm{~A}$ magnetic drug delivery composite was designed with doxorubicin (DOX) attached to $\mathrm{Fe}_{3} \mathrm{O}_{4}$ nanoparticles chemically. ${ }^{138}$ They are then immersed in polyethylene glycol (PEG) stabilized porous silica shell $\left(\mathrm{Fe}_{3} \mathrm{O}_{4}-\mathrm{DOX} / \mathrm{pSiO}_{2}-\mathrm{PEG}\right)$. A protective coat of porous silica gel present on drug molecules surface also acts as a thin obstruction for DOX escape from the composite carrier.

Eudragit is a $\mathrm{pH}$-dependent polymer frequently used for the coating of colon-targeted drugs for the delivery of drugs and can be regulated to transport a drug at any other target site. ${ }^{139}$ Superparamagnetic nanoparticles coated with Eudragit, encapsulating doxorubicin produced by double emulsion technique using ethanol as a solvent were investigated for in-vitro drug release studies. ${ }^{87}$ These colloidal particles showed initially sustained releases followed by the slow and exponential release of drug by sustaining a therapeutic level for a longer period relative to an unbounded drug. IONPs coated with aminosilane were inoculated in an intravenous tumor model of rats and then increasing the temperature of a tumor to about $43{ }^{\circ} \mathrm{C}$ initiating tumor 
reversion in an alternating magnetic field. ${ }^{140}$ Zhao and co-workers witnessed that in an alternating magnetic field, introducing IONPs solution directly into head and neck cancer cells of mice died in 30 minutes, did not affect normal cells. ${ }^{141}$ Besides, this complex was permitted to slip away the reticuloendothelial system by biocompatible polymer PEG, hence permitting the drugs to be supervised over extended periods. Poly (N-isopropyl acrylamide) enclosing $\mathrm{Fe}_{3} \mathrm{O}_{4}$ magnetic nanostructure (MNS) was also present in which DOX was filled into the hydrogel via absorption. Due to the thermal activation of MNS, drug release in an RF field was found to be two times greater than in the absence of the radiofrequency field (RF) field. In the test tube, local drug transport of the DOX-filled hydrogel-MNS composite with HeLa cell lines showed that more than $80 \%$ cells were dead in RF field while $40 \%$ cells were dead without RF field. ${ }^{142}$ MNPs encapsulated inside the polycaprolactone (PCL) microsphere along with ciprofloxacin were used as media to initiate the drug release immediately by magnetic stimulation. ${ }^{143}$ A single emulsion method was utilized to develop a novel nanocarrier, containing magnetite nanoparticles with doxorubicin inside PLGA (poly (lactic-co-glycolic acid)) biocompatible shell. They were used for intratumoral administration to release a drug in a controlled manner. ${ }^{144}$ Microbubbles were also prepared by encapsulating $\mathrm{Fe}_{3} \mathrm{O}_{4}$ nanoparticles and doxorubicin as multifunctional theranostic agents that deliver a plan for imaging and chemotherapy of primary tumors. ${ }^{145}$ Dual anticancer theranostic agents were developed by loading paclitaxel (PTX) and SPIONs inside PLGA nanoparticles for imaging and cancer treatment by monitoring drug release. ${ }^{146}$ Doxorubicin-loaded magnetite nanoparticles were prepared and modified with PLGA-PEG copolymer through a double emulsion method. These polymer-drug composite nanoparticles are used as a controlled drug release system. ${ }^{147} 5$ (fluorouracil) FU-encapsulated MNPs modified with biocompatible copolymers PCL-PEG-PCL by double emulsion technique, was used as a carrier for release of drugs in a controlled mode for in vivo as well as in vitro investigation. ${ }^{148}$ Superparamagnetic magnetite nanoparticles with oleic acid coating are encapsulated in uniform size PLGA nanospheres by a modified double emulsion method as a diagnostic and therapeutic agent for MRI studies. ${ }^{149}$ PEGylated curcumin was utilized to physically decorate magnetic nanoparticles surface to form composites MNP@PEG-Cur to instantaneously get benefit from magnetic targeting characteristics of nanocomposites. The nanoparticles indicated the release of the drug depending on $\mathrm{pH}$, with more release inside the acidic medium $(\mathrm{pH}=5.4)$. Also, hemolysis assay and cell viability established MNP@PEG-Cur biocompatibility in physiologic 
pH close to 7.4. ${ }^{150}$ As an innovative drug delivery system, methotrexate (MTX) was bonded to iron oxide MNPs with arginine capping, utilized as a vehicle in delivery of the drug in a controlled way and as a contrast agent in MRI. Certainly, no cytotoxicity was shown by the prepared nanoparticles to cell lines of MCF-7, 4T1, and HFF-2. ${ }^{151}$ The targeted drug delivery structure was developed by stabilization of magnetic $\mathrm{Fe}_{3} \mathrm{O}_{4}$ nanoparticles using chitosan and Imatinib. $\mathrm{Fe}_{3} \mathrm{O}_{4} @ \mathrm{CS}$ acts like a pH-responsive nanocarrier to release Imatinib molecules and unveiled a cytotoxic effect against breast cancer MCF-7 cells. It is an efficient pH-responsive delivery system advantageous to conventional chemotherapy. ${ }^{152}$ Silica-coated iron oxide nanoparticles act as a very good dopamine delivery agent to kill cancer cells. $\mathrm{Fe}_{3} \mathrm{O}_{4}$ nanoparticles loaded with dopamine (DA) and coated with silicon dioxide $\left(\mathrm{SiO}_{2}\right)$ showed a substantial enhancement in the release of drug in the acid medium compared to the typical $\mathrm{pH}$ of $7.4 .{ }^{153}$ The availability of anticancerous, slightly water-soluble drug camptothecin (CPT) became upgraded through the incorporation of MNPs with it. The drug incorporation with magnetic nanoparticles in a composite allows the controlled drug release used to study cancer-fighting in live creature's bodies. ${ }^{154}$ Thus, magnetic nanoparticles are used as an effective carrier system with attractive biomedicine and biotechnology applications.

Poly(lactide-co-glycolide) (PLGA) nanocomposite particles containing 5-fluorouracil (5-FU) and gold-decorated magnetite nanoparticles with a raspberry-like morphology were developed as multidisciplinary probes in drug delivery systems and cancer therapy with high performance and minimum side effects. With the coexistence of gold and $\mathrm{Fe}_{3} \mathrm{O}_{4}$ nanoparticles, the final probe provided enhanced dual magneto and photothermal responses. The drug release profile of the 5FU-loaded nanocomposite particles demonstrated their controlled release at $37{ }^{\circ} \mathrm{C}$ in phosphatebuffered saline solution and indicated their potential in cancer therapy by photothermal to treat DU145 prostate cancer cells. ${ }^{155}$ However the biodegradability and safety risks of photoresponsive magnetic composites are still questionable. ${ }^{156}$ Additionally, UV light cannot penetrate tissues deeper than $\sim 10 \mathrm{~mm}$ due to its absorption by endogenous chromophores such as oxy- and deoxy-hemoglobin, lipids, and water; and prolonged UV irradiation can be cytotoxic. Therefore light-responsive nanotherapeutics should be used restrictively to the eye, skin, and other mucosa surfaces. ${ }^{157}$ The novel dual-stimuli-responsive nanocomposites based on silica-coated iron oxide/polyaniline (Si-MNPs/PANI) have also been developed for biomedical applications. Results showed that Si-MNPs/PANI nanocomposites displayed both magnetically 
and electrically-responsive characteristics. Moreover, in vitro cytocompatibility and hemocompatibility of these composites suggesting their use in certain doses for controlled-drug delivery. ${ }^{158}$ Despite their flexibility and low-cost advantages, there is a risk of damage to healthy tissue from an electric source due to deep tissue penetration (attenuation of a stimulus) using electrical stimulus, which limited their use for biomedical applications. ${ }^{156}$

Magnetic Nanoparticles for Magnetic Separation and Purification. Magnetic separation offers exceptional prospects in the field of biomedicine with sensitive, selective, and target capture in a controlled manner. Isolation and purification of bioanalytes including enzymes, nucleic acids, antibodies, and proteins depend on complex, lengthy, chromatographic and electrophoretic procedures that involve multiple steps. These methods involve the interaction of biomolecules of interest, immobilized on solid matrix. An alternative to these techniques is to utilize MNPs conjugated with affinity ligands to separate biomolecules from the mixture in respect of labor, time, and yield. An ideal and competent platform for separation having high magnetophoretic mobility is magnetically reactive. They also react fast to the magnetic field externally applied, and this characteristic is influenced by the magnetic susceptibility and size of materials. ${ }^{159}$ A schematic representation of several steps of bioseparation of bioanalytes bound to MNPs using a magnetic field is presented in Figure 6. 


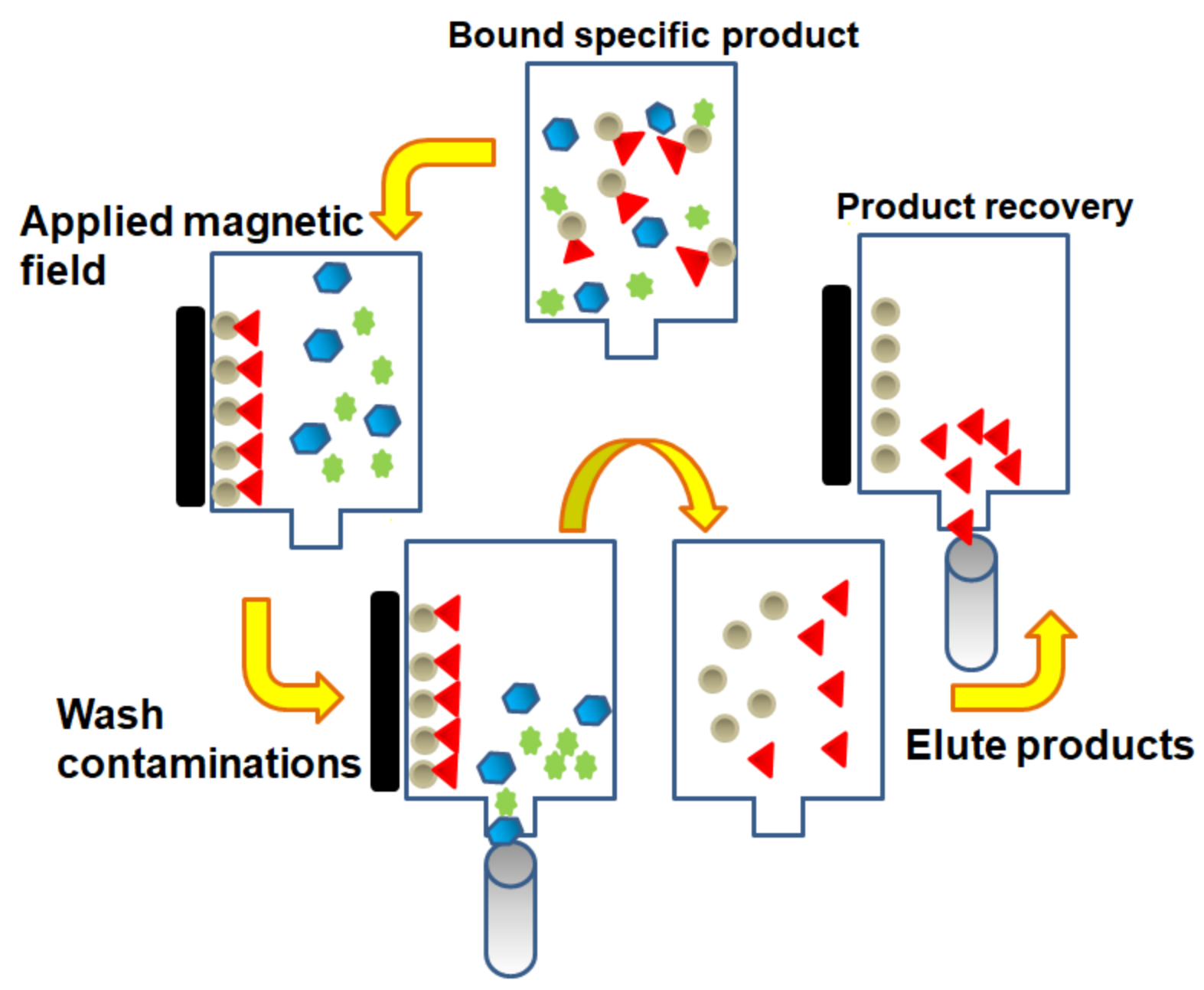

Figure 6. Representation of bioseparation using magnetic nanoparticles. (The bioanalytes of interest conjugated to MNPs are pumped into separated column controlled by a magnetic field having a filter. The first step is to retain the conjugated bioanalytes and elimination of contaminants by switching on the magnetic field. Then unbounded bioanalytes are eliminated by fresh washing buffer solutions entering column and through continuous switching on and off of magnetic field. The particles are recollected from the washing buffer and recovered by passing elution buffer with magnetic field off. Finally, the eluted product is purified with the help of magnetic field).

Currently, MNPs have been widely utilized in bioseparation because of their stability, good biocompatibility, magnetic characteristics, and high binding capacity. MNPs separate biological analytes from raw specimens, and lessen the non-specific adsorption of interfering bioanalytes lacking any damage to sample. ${ }^{160}$ MNPs are highly suitable for separation purposes due to their low cost and have high removal capacity, reusability, high surface-volume ratio, and reactivity 
towards eluent compounds. It is also efficient than filtration and centrifugation. Due to the poor solubility of MNPs, they are modified with various kinds of materials to protect their outer surface from strong $\mathrm{pH}$, high temperature for successful immobilization and separation of protein, nucleic acid, antibodies, enzymes, targeting agents, and additional biomolecules. ${ }^{161}$

A novel system was developed PNIPAAm functionalized gold $(\mathrm{Au})$ and magnetite $\left(\mathrm{Fe}_{3} \mathrm{O}_{4}\right)$ nanoparticles for separation of streptavidin (model protein) from human plasma. Gold nanoparticles functionalized with positively charged PNIPAAm carry biotin, an affinity ligand, against streptavidin. PNIPAAm was used as a stabilizer ligand in the synthesis of $\mathrm{Fe}_{3} \mathrm{O}_{4}$ nanoparticles. Aggregation of particles is caused by the incubation of Au-PNIPAAmbiotin and $\mathrm{Fe}_{3} \mathrm{O}_{4}$-PNIPAAm along with streptavidin spiky plasma at the temperature of 45 ${ }^{\circ} \mathrm{C}$. Au-PNIPAAm-biotin attached streptavidin became concentrated utilizing magnetic separation and quantified into smaller volume devoid of any extra handling with lateral flow immuno-chromatography test. ${ }^{162}$ Magnetic separation was performed for efficient islet cell separation using dextran, heparin, or siloxane-coated SPIONs. The labeling of islets by SPIONs remains advantageous in islet separation for transplant purification and also for in vivo imaging next to transplantation. ${ }^{163}$ The preformed silica-coated $\mathrm{Fe}_{3} \mathrm{O}_{4}$ nanoparticles with amine terminal groups bonded with antibody (anti-CD133) are particular to cluster of variation biomarkers (CD133) in neural stem cells. They were vaccinated into the region of cerebrospinal fluid of the brain of a rat in the magnetic field, externally applied. Stem cells from the endothelial lining along with the MNPs were released and collected with a syringe or by a magnet probe. ${ }^{164}$ Protein A coated magnetic nanoparticles are exploited in preparatory refinement of antibodies (IgGs) from a cell culture. ${ }^{165}$ DNA adsorption and desorption mechanism on temperaturesensitive PNIPAM-co-AEMH (poly (N-isopropylacrylamide-co-aminoethylmethacrylate)) coated magnetic nanoparticles were consumed in separation microsystem for extracting, refining, and concentrating nucleic acid. These dual responsive particles were used as rigid support in the separation of DNA from biological blends for diagnosis that offer pronounced potential for emerging separating versatile tools. ${ }^{166}$

The separation techniques based on MNPs have become repetitive quantitative and qualitative tools for diagnosis in biomedical applications. Integrated with additional detection methods like microscopy, magnetic separation becomes a fast, appropriate, careful, and delicate method for decontamination, enhancement, and detection of cells and biomolecules. It can also be 
engineered as an essential part of microfluidic devices. In the future, MNPs can be used for in vivo biomolecules separations for diagnostics and removal of pathogens, tumors, antigens locally or systemically.

Magnetic Nanoparticles for Chemotherapy. Chemotherapeutic treatment mostly used to treat cancer mainly to kill tumor cells has no abilities of specific cell-targeting and possess cytostatic and cytotoxic effects. MNPs possess the ability to be tailored for targeting a particular site after internalization. These nanoparticles are loaded with therapeutic compounds that were released to the cytoplasm of cell to perform the preferred function. The therapeutic compound is formed by conjugation of chemotherapeutic drug agents with MNPs that are inoculated into blood and operated by magnetic field externally applied to target specific tumor site. ${ }^{167}$ The chemotherapeutic remedies start with the release of drug to destroy the cellular structure of cancer cells. Impact of magnetic field on MNPs for transportation of drug safely to the internal microenvironment and also enhances the biodistribution. MNPs permit the usage of therapeutics at sufficiently low doses which consequently decreases the toxic effect of drugs. These therapeutic compounds have many benefits over orthodox for drug delivery systems like an increase in hydrophobic solubility, enhancement in intracellular infiltration, and long drug circulation time. ${ }^{168}$ Controlled delivery systems for drugs reduce the nonspecific uptake, have better targeting ability, controlled drug release, more operative, and provides suitable routes of administration (pulmonary, oral, transdermal, and parenteral). ${ }^{168}$ The engineered nanocarrier based on IONPs functioned as a real and operative transport carrier for the drug, doxorubicin. It could kill cancer cells distantly by generating heat in an AMF (alternating magnetic field). The prepared polyethylene glycol (PEG) coated nanocarrier functionalized with Luteinizing Hormone-Releasing Hormones (LHRH) peptide increases their stability in physiological media and makes them able to target cancerous cells. ${ }^{169}$ Thus the developed IONPs-based system is robust for ovarian cancer treatment by combinatorial approach as shown in Figure 7. 


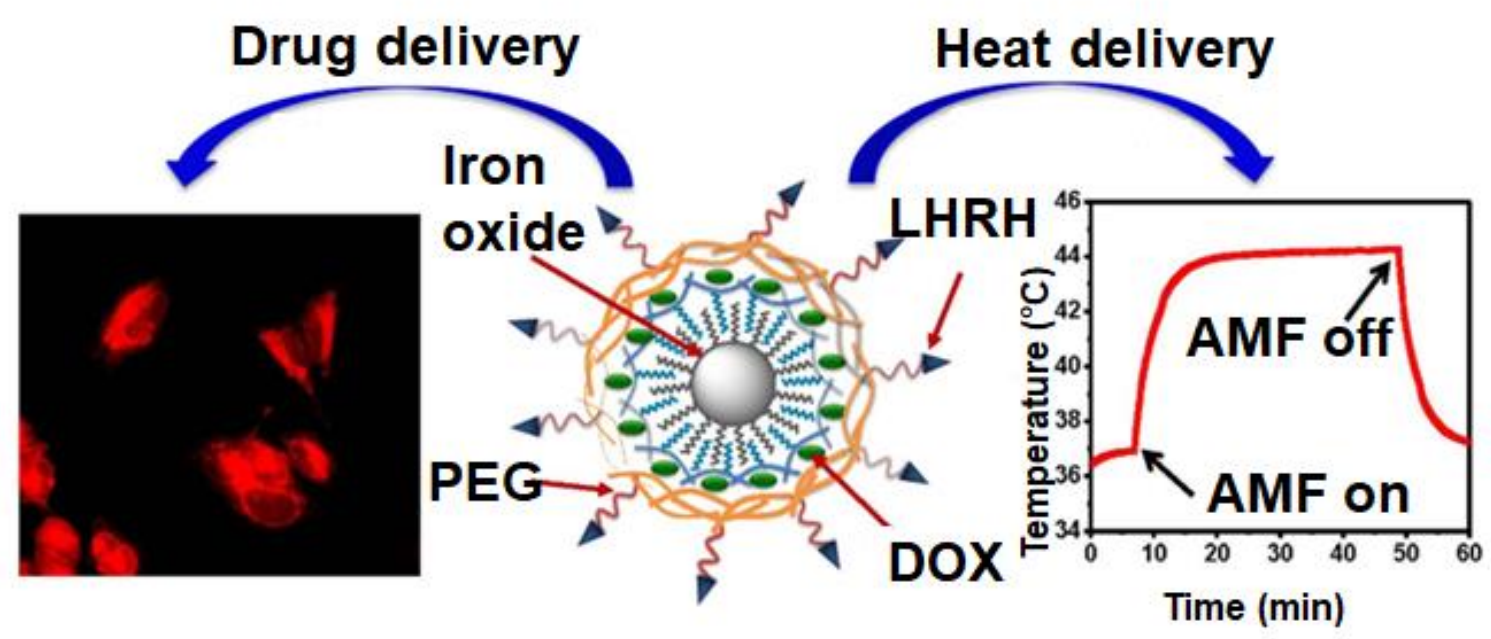

Figure 7. Nanocarrier for combined chemotherapy and hyperthermia due to the release of therapeutic drug and heat respectively. Reprinted from ref ${ }^{169}$. Copyright 2020 Elsevier. A multifunctional magnetic-based nanocarrier as a proficient doxorubicin delivery vehicle has also the ability to kill cancer cells in an AMF. The prepared nanocarrier verified the rapid release of drug in an acidic medium that mimic tumor surroundings. Mild hyperthermia $\left(40{ }^{\circ} \mathrm{C}\right)$ produced by the developed nanocarrier in AMF synergistically enhances the cytotoxicity of doxorubicin delivered to cancer cells.

A delivery system for the model drug was developed from Doxorubicin-loaded magnetic alginate-chitosan microspheres for breast cancer (MCF-7) treatment. Magnetic hyperthermia agents (SPIONs) were used as to stimulate drug release, doxorubicin. MCF-7 cells exhibited substantial cell death in vitro analysis as compared to only chemotherapy or magnetic hyperthermia. In vivo investigations of antitumor showed the disappearance of remaining tumor in twelve days when combined with chemo-thermal treatment. ${ }^{170}$ The polyethyleneiminefunctionalized graphene oxide (GO)-coated $\mathrm{Fe}_{3} \mathrm{O}_{4}$ nanospheres, loaded with doxorubicin, were developed to investigate the tumor-cell-killing efficiency. In vivo as well as in vitro investigations proposed a system (DOX-GO/IONP/PEI) for drug release that effectively caused the death of tumor cells due to chemotherapeutic agent effects, without adversely affecting surrounding healthy tissues. ${ }^{171}$ Doxorubicin has also been encapsulated inside microspheres consisting of poly (lactic-co-glycolic acid) (PLGA) coupled with further functionalized by $\gamma$ $\mathrm{Fe}_{2} \mathrm{O}_{3}$ nanoparticles for the examination of its ability to inhibit tumor growth. The MNPs inside the shell induced both the heat effect and enhance the permeability of the shell to release drugs. The hyperthermia coupled with chemotherapy is favorable for in vitro breast cancer cell (4T1) 
apoptosis. ${ }^{172}$ MNPs and carmustine (lipophilic drug) were loaded in the lipid core of oleosome for cancer treatment. The burst release of the drug from these magnetic oleosomes in magnetic field leads to anti-cancer activity. ${ }^{173}$ Combinatorial therapies including thermotherapeutic and chemotherapeutic effects have an imperative role in treating local pancreatic cancer treatments (BxPC3 and PANC-1). The incorporation of Gemcitabine and/or NucAnt (N6L) with MNPs resulted in a controlled release of chemotherapeutics in a magnetic field due to generation of heat, locally, that strongly inhibits cell growth. ${ }^{174} \mathrm{~A}$ silica-coated magnetic nanoparticle multifunctional system was synthesized encapsulating a chemotherapeutic agent having magnetic core, coated with specific "Heat Shock Protein Inhibitor" against the lung cancer stem cells (CSCs). These nanoparticles were administered systematically for combined chemotherapy and thermotherapy utilising alternating magnetic field applied externally. Lung CSCs were killed up to $98 \%$ in vitro within 30-min because of combined chemotherapeutic drug and hyperthermia treatment. This collective therapy considerably inhibited metastasis and growth of tumor in lung CSC xenograft-bearing mice in vivo models, having negligible side effects. ${ }^{175}$ Novel hydrogel consisting of SPIONs and loaded with doxorubicin showed a controlled drug release pattern and sustainable therapeutic ability. This unique hydrogel formulation showed firm potential for prevention of relapses of numerous cancers. ${ }^{176}$

Nanoparticles, with $\mathrm{Fe}_{3} \mathrm{O}_{4}$ core, in an alternating magnetic field have ability to produce sufficient heat for active hyperthermia in corporation with chemotherapy by the doxorubicin release of from alginate shell. Folate was bonded to these nanoparticles that aid the developed nanocomposite to pass in and remain in tumor cells for longer period of time. In vivo experimentation revealed the practicality of mutual treatment for lung cancer in mice, particularly because of the presence of folate factor. ${ }^{177}$

\section{Magnetic Nanoparticles for Gene Therapy and Enzyme Immobilization. A delivery} platform, comprising of MNPs incorporated with gene, is a favorable approach for treating both genetic and acquired human diseases. It involves MNPs contain viral, polymeric, and non-viral platforms that enhance its cellular uptake to treat genetic diseases. ${ }^{178}$ The main idea of the proposed therapeutic system is the introduction of an exogenous gene containing with a functioning gene to encode the practical protein to treat the disease-causing mutation. These platforms are advantageous concerning gene delivery like the capability of particular tissue or 
cells targeting, improve DNA stability, protection of target gene from nuclease degradation, and intensify the transformation effectiveness or care. ${ }^{179}$ Gene therapeutics along with MNPs was assimilated as gene vectors for the preservation of nucleic acids against enzymatic deterioration and for endosomal deliverance subsequently after cellular internalization. ${ }^{178}$

Magnetofection is the integration of MNPs with gene vectors for the improvement of gene transfer in a magnetic field. Gene therapy is used in genetic deficits and additionally other complex illnesses, like viral infection, autoimmunity (rheumatoid arthritis), artery and coronary diseases, diabetes, neurodegenerative conditions, asthma, AIDS, and uncountable genetic diseases which effect humanity. ${ }^{180}$ Magnetic nanoparticle-based gene transfection improves in vitro complete transfection levels in human bronchial epithelial cells. A novel DNA nanovector was formed by the covalent bonding of DNA on naked MNPs directly resulted in a tremendously robust gene delivery system. It was liable for the improved cellular uptake and enhanced the transfection efficiency in mesenchymal stem cells. MNPs were conjugated with therapeutic gene carriers to overcome the poor targeting of conventional gene therapy protocols. ${ }^{181}$ MNPs with diverse surface variations can boost hemagglutinating virus Japan envelope (HVJ-E) -based gene transfer through adjustment of charge with size, that potentially assist in overcoming essential restrictions to in vivo gene therapy. A combination of magnetite particles and protamine sulfate considerably improved transfection efficiency in an in vitro cell culture system consisting of BHK21 cells, irrespective of decrease in quantity of HVJ-E. ${ }^{182}$ PEI modified MNPs gene delivery systems increases concentration of DNA and prevents its degradation. MNPs can efficiently deliver the exogenous gene inside somatic cells of mammal with extremely constant gene expressions demonstrated by intracellular tracking. ${ }^{183}$ Thus magnetically-guided targeting of gene by MNPs is promising technique for transfection of multiple tissue explants and cell lines for gene therapy. Theranostic MNPs encapsulated within polymers exhibit imaging properties and can efficiently deliver numerous genes for therapeutic purposes (Figure 8). Polysaccharide-based MNPs were fabricated for magnetofection-based gene delivery systems were favorable contestants for playing roles as therapeutic agents and as imaging contrast agents. They can further be bioconjugated with agents for cell-targeting. ${ }^{184}$ 


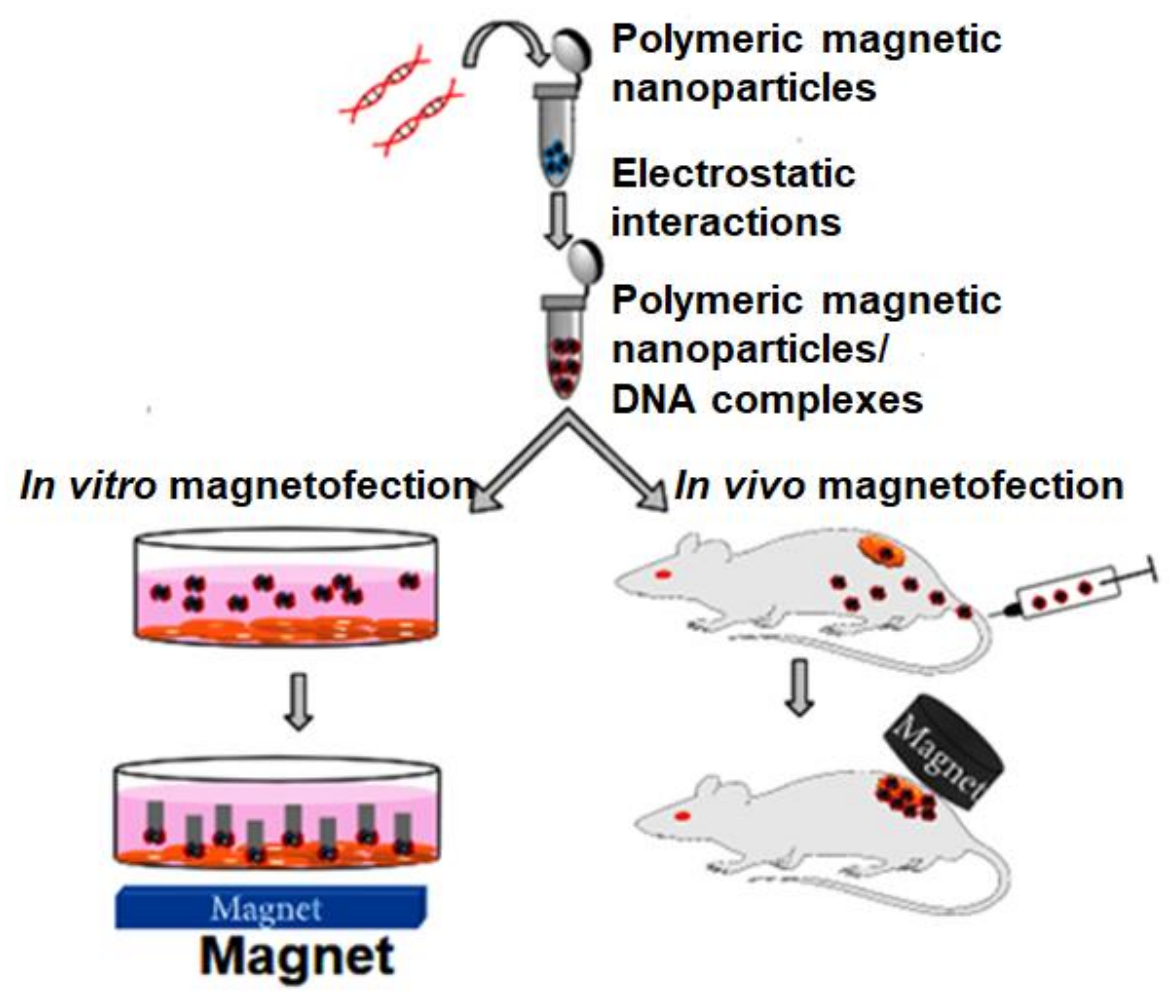

Figure 8. Illustration of in vivo and in vitro delivery of gene by magnetofection (on the left corner of the image, grey color patterns signifies the path of MNPs movement due to the effect of a magnet). Reprinted from ref ${ }^{184}$. Copyright 2020 Hindwai.

MNPs were also used to design an efficient enzyme delivery system to control the mechanisms of cell-specification, suitable in clinical treatment. MNPs modified with a reactive biocompatible polymer, poly (2-vinyl-4,4-dimethyl azlactone) acts as appropriate carriers for immobilization of enzyme L.Asparaginase. The prepared enzyme L.Asparaginase reactor has long-term stability and potential capability to treat acute lymphoblastic leukemia. ${ }^{185}$ Serratiopeptidase, a therapeutic enzyme intended for inflammation and pain was covalently immobilized on chitosan amino-stabilized MNPs by glutaraldehyde. The prepared conjugates showed enhanced anti-inflammatory effect in vivo in rats. ${ }^{186}$ The magnetic $\left(\mathrm{Fe}_{3} \mathrm{O}_{4}\right)$ nanoparticles functionalized with alkyl trimethoxy silane having proper alkyl length were exceptional candidates as operative carriers for immobilization of lipase for effectual recycling and recovery of an enzyme. ${ }^{187}$ Trypsin enzyme immobilized on magnetite nanoparticles through noncovalent bonding to preserve the enzyme activity for successive reuse. ${ }^{188}$ MNPs have been conjugated with biotin and (strept)avidin for protein immobilization that significantly caused the reduction of the risk of denaturation or degradation of protein. ${ }^{189}$ First, magnetic nanoparticles were 
prepared by chemical coprecipitation technique, and functionalized with protein fluorescien isothiocynate (FITC)-labelled streptavidin by carbodiimide activation. Next, gold-coated glass slides were modified with biotin self-assembled monolayers of biotin-HPDP. Schematic of this process is shown in Figure 9. In the next step, the modified nanoparticles precisely bound to biotinylated gold surfaces which were used for biosensing.

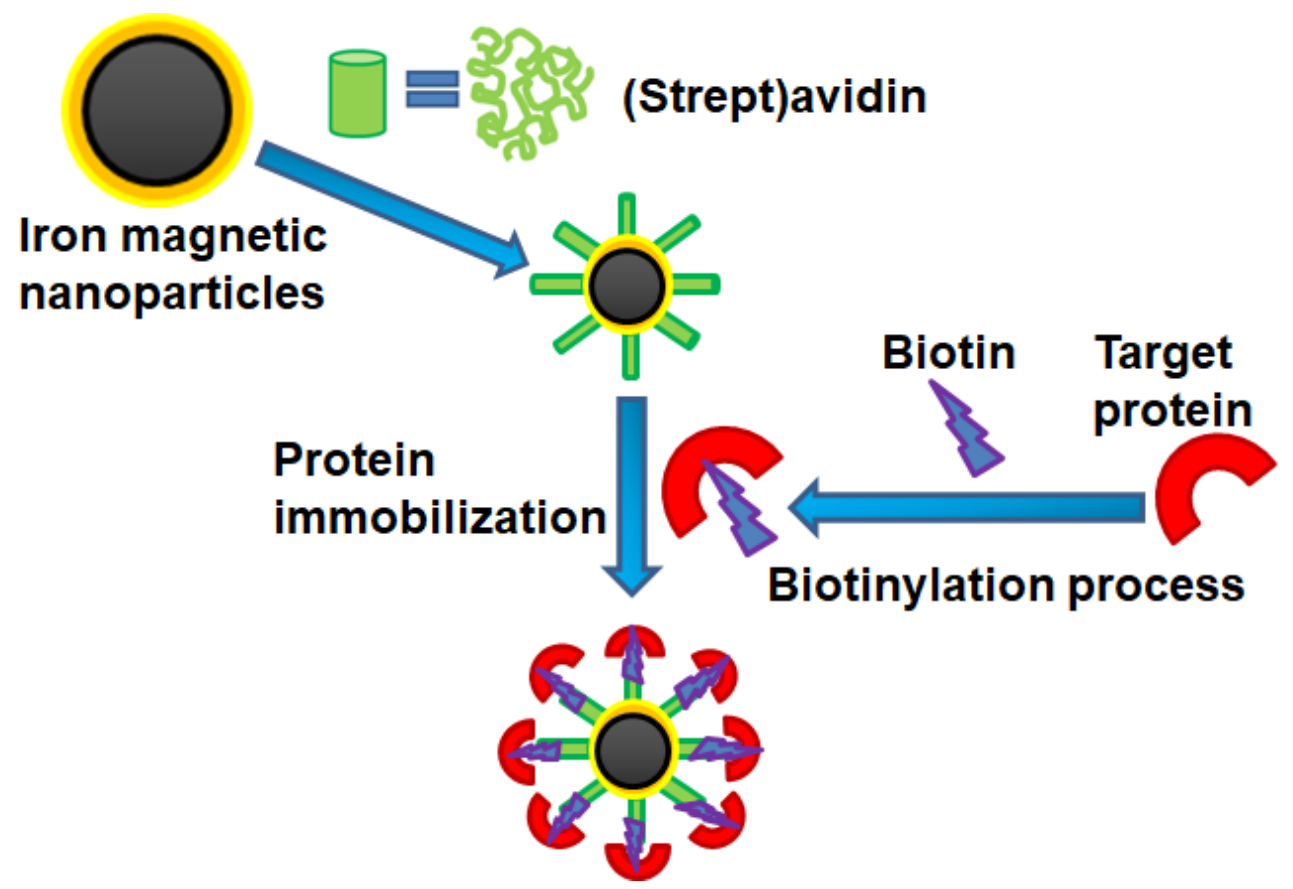

Figure 9. Illustration of immobilization of protein over magnetic nanoparticles for biological recognition using (strept)avidin-biotin technology. MNPs were first functionalized with protein fluorescien isothiocynate FITC-labelled streptavidin and goldcoated glass slides were modified with biotin self-assembled monolayers of biotin-HPDP. Then modified nanoparticles precisely bound to biotinylated gold surfaces which were used for biosensing.

MNPs have developed as adaptable and multipurpose tool to create exceptional supports for immobilization of enzyme because of large surface area and small size. Different MNPs have been synthesized with appropriate surface functionalization, and successfully employed for immobilization of enzyme/protein, that already showed promising properties in many real-world applied bioapplications.

Magnetic Nanoparticles for Photothermal Therapy (PTT). Magnetic nanoparticles are a good alternative for use as a photothermal agent in the treatment of tumors (Figure 10). Such 
nanoparticles are used in combination with other substances or used themselves as photothermal agents. The combination of magnetic nanoparticles with other photothermal agents adds more capabilities to the therapeutic system. Among the magnetic particles with dual functions of magnetism and NIR absorption, iron oxide nanoparticles stand out as nanoparticles suitable for PTT. They have great potential for use in biomedical applications because they can be directed magnetically to the site of interest (the tumor) and their distribution in tumors and other organs can be imaged. ${ }^{190}$

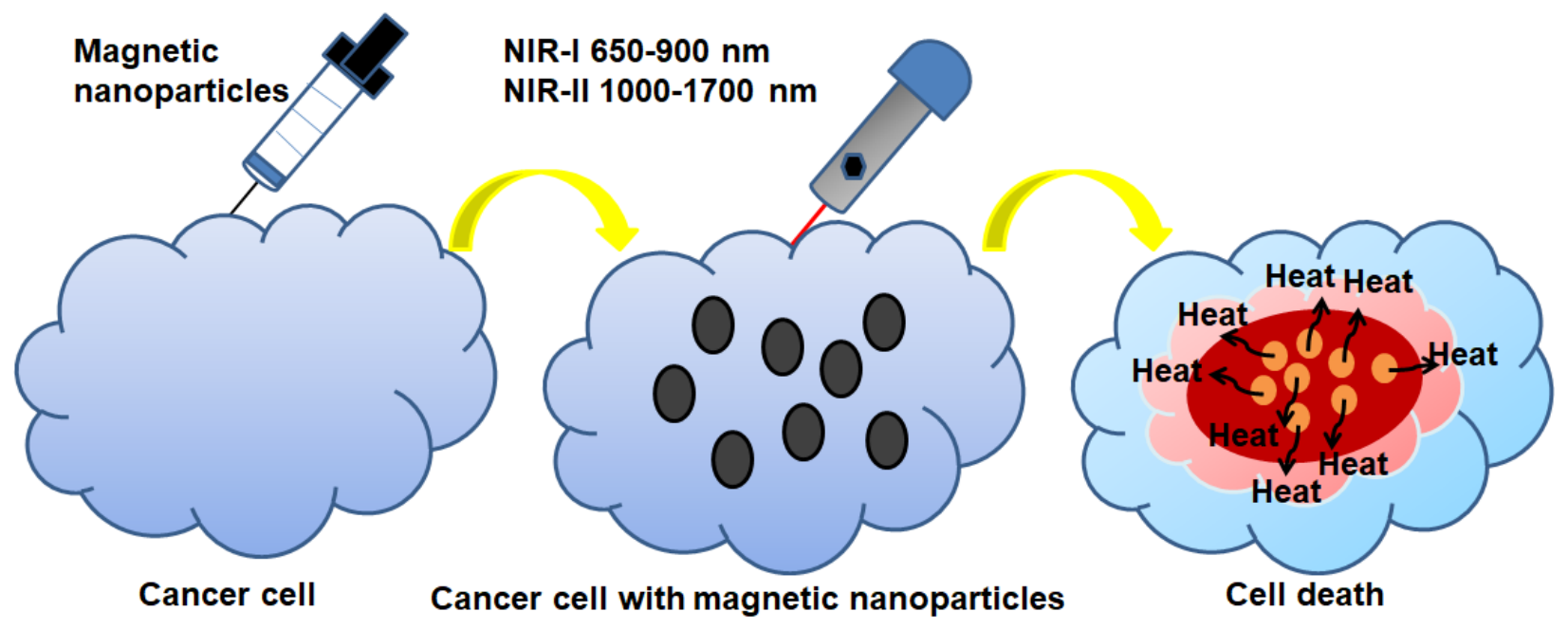

Figure 10. Schematic illustration of cell death by injecting nanoparticles as photothermal agent for the treatment of tumor cells.

The combination of an alternating magnetic field and infrared absorption into a single composite will make it possible for the nanoparticles to be guided until the tumor. They can also work in MRI for visualizing the location of cancer, or in thermal imaging for real-time monitoring of the treatment. This would also increase the heating efficiency of iron oxide nanoparticles. ${ }^{191}$

For effectively treating tumor related-bone defects, MNPs modified-porous scaffold was reported firstly for effectively treating tumor-induced bone defects. Fabricated biomaterials consisting of $\mathrm{SrFe}_{12} \mathrm{O}_{19}$ nanoparticles modified-mesoporous bioglass (BG)/chitosan (CS) porous scaffold (MBCS) showed excellent bone regeneration and antitumor function. The $\mathrm{SrFe}_{12} \mathrm{O}_{19}$ nanoparticles in MBCS have improved the photothermal conversion property under the irradiation of near-infrared (NIR) laser. The elevated temperatures of tumors co-cultured with MBCS triggered tumor apoptosis and ablation. Thus these magnetic nanoparticles in the scaffold not only accelerated the stem cell proliferation, osteogenic differentiation and new bone regeneration, but also improved photothermal therapy efficacy against bone tumors. ${ }^{192}$ For the 
first time a platelet-cancer stem cell (CSC) hybrid membrane-coated iron oxide magnetic nanoparticle [CSC-P] MNPs has been presented, e for the enhanced photothermal therapy of head and neck squamous cell carcinoma (HNSCC). The [CSC-P] MNPs possess superior characteristics for immune evasion, active cancer targeting, magnetic resonance imaging, and photothermal therapy. As compared to single cell membrane-coated MNs, [CSC-P] MNPs, they exhibit prolonged circulation times and enhanced targeting abilities. Their photothermal ability provides inhibits HNSCC tumor growth, particularly in an immunocompetent Tgfbr1/Pten conditional double knockout HNSCC mouse model that is parallel to the human HNSCC microenvironment. Together, this biomimetic multimembrane-coated nanoplatform enhanced antitumor the efficacy in the complex tumor microenvironment. ${ }^{193}$

The core-shell $\mathrm{Fe}_{3} \mathrm{O}_{4} @ \mathrm{Au}$ magnetic nanoparticles (MNPs) were prepared, further $\mathrm{C} 225$ was absorbed to fabricate $\mathrm{Fe}_{3} \mathrm{O}_{4} @ \mathrm{Au}-\mathrm{C} 225$ composite-targeted MNPs. After characterizations, the glioma-destructive effect of magnetic fluid hyperthermia (MFH) united with near-infrared (NIR) hyperthermia mediated by $\mathrm{Fe}_{3} \mathrm{O}_{4} @ \mathrm{Au}-\mathrm{C} 225$ composite-targeted MNPs was estimated by in vitro and in vivo experiments. The inhibitory and apoptotic rates of $\mathrm{Fe}_{3} \mathrm{O}_{4} @ \mathrm{Au}-\mathrm{C} 225$ compositetargeted MNPs-mediated combined hyperthermia (MFH+NIR) group were significantly higher and suppress the tumor growth compared with other groups in vitro and in vivo. $\mathrm{Fe}_{3} \mathrm{O}_{4} @ \mathrm{Au}-$ C225 composite-targeted MNPs have great potential as a promising nanoplatform for human glioma therapy and could be of great value in future for medical use. ${ }^{194}$ Poly(L-lactic-coglycolic acid) nanoparticles were loaded with oleic acid- $\mathrm{Fe}_{3} \mathrm{O}_{4}$ and triphenylamine-divinylanthracenedicyano further modified by anti-VEGF antibodies to form anti-VEGF/OAFe $\mathrm{O}_{4} /$ triphenylaminedivinylanthracene-dicyano@poly(L- lactic-co-glycolic acid) NPs. This system has capability to recognize the overexpressed VEGF-A as low as $68 \mathrm{pg} / \mathrm{ml}$ in different cell lines with good selectivity and photothermal therapy effect. These ultrasensitive theranostic nanoparticles were able to identify tumor cells by fluorescence imaging and MRI, and destroy tumors under near infrared illumination. ${ }^{195}$

A magnetic nanoparticle delivery system was constructed by the combination of photothermal therapy (PTT) with immunotherapy. This delivery system is consisted of $\mathrm{Fe}_{3} \mathrm{O}_{4}$ magnetic nanoparticles (MPs) as the core to load indocyanine green (ICG) and coating of polyethylene glycol polyphenols (DPA-PEG) to load R837 hydrochloride (R837), that formed R837 loaded polyphenols coating ICG loaded magnetic nanoparticles (MIRDs) which provides an effective 
strategy in cancer treatment (Figure 11). Afterward intravenous injection, results showed long circulation, magnetic resonance imaging (MRI) guides, and magnetic targeting. Once targeting to the tumor, the MIRDs with the near-infrared (NIR) irradiation triggered tumor ablation and resulted in tumor-associated antigens releasing to induce the body's immunological response, which was markedly improved it to attack the tumors with the R837 releasing from the outer DPA-PEG. The combination of the PTT and immunotherapy inhibited tumor growth and caused potent anticancer therapeutic effects with few side effect. ${ }^{196}$

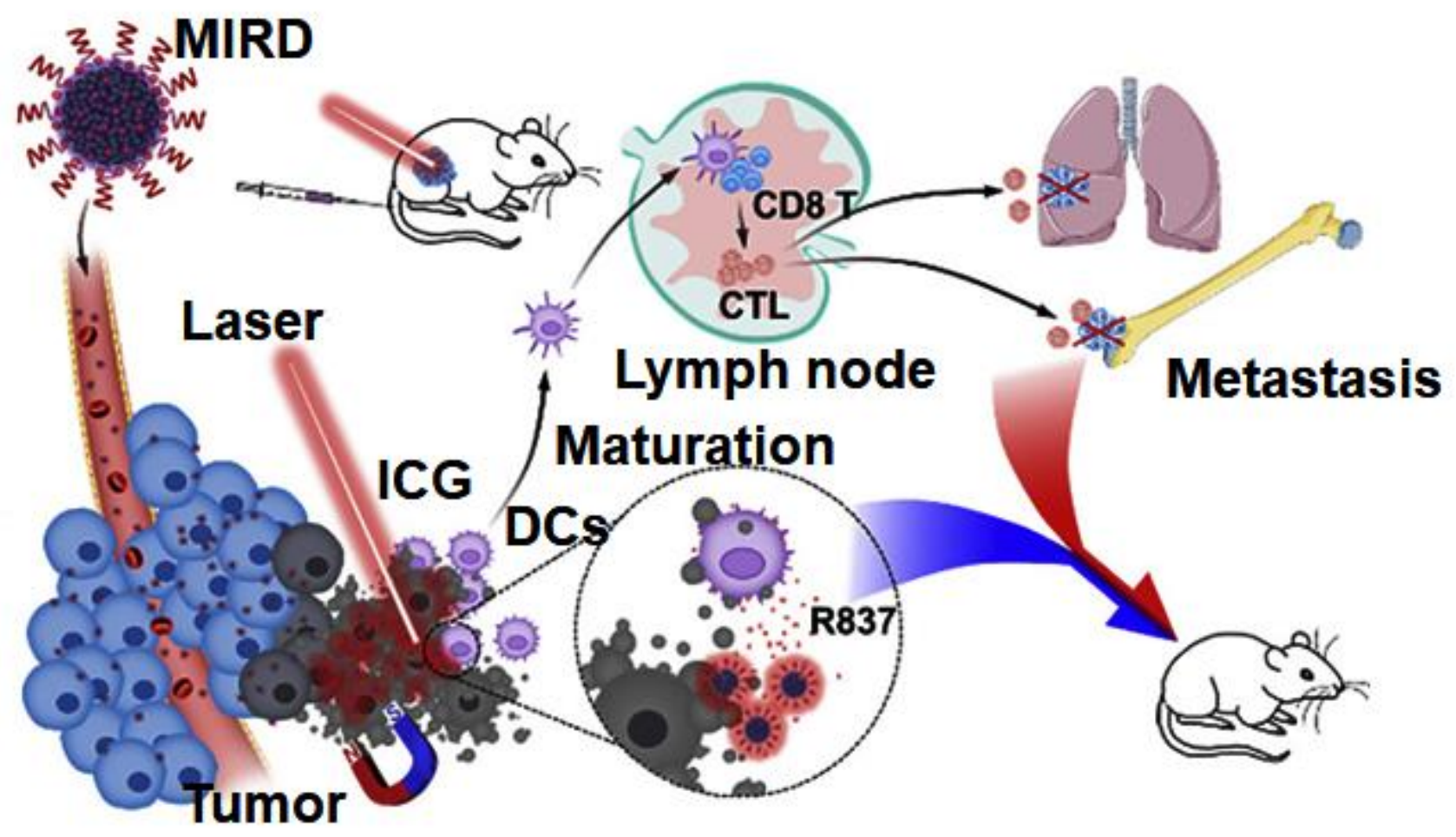

Figure 11. Magnetic nanoparticles coated with polyphenols for spatio-temporally controlled cancer photothermal/immunotherapy. A magnetic nanoparticle is used as a carrier for magnetic tumor targeting and provides a visible treatment by MRI. Magnetic accumulation of ICG in tumor and controlled release of R837 enhances the PTT effect. Once targeting to the tumor, the MIRDs with the near-infrared (NIR) irradiation triggered tumor ablation and resulted in tumor-associated antigens releasing to induce the body's immunological response, which was markedly improved it to attack the tumors with the R837 releasing from the outer DPA-PEG.Reprinted from ref ${ }^{196}$. Copyright 2021 Elsevier. Multifunctional nanocarriers based on PAMAM dendrimers fixed to polydopamine (PDA) coated magnetite nanoparticles $\left(\mathrm{Fe}_{3} \mathrm{O}_{4}\right)$ were successfully applied to combined chemo- and 
photothermal therapy (CT-PTT) of the liver cancer cells. The supportive effect of the modalities mentioned above led to higher mortality of cancer cells as compared to their individual performance. The in vitro studies revealed that the application of dual therapy triggered the desired cell death mechanism-apoptosis. Additionally, performed tests using Magnetic Resonance Imaging (MRI) indicated that our materials have reasonable contrast properties. The functionality of dendrimers has been extended by merging them with magnetic nanoparticles resulting in multifunctional hybrid nanostructures that are promising smart drug delivery system for cancer therapy. ${ }^{197}$

Thus MNPs appear as promising photothermal agents because of excellent magnetic properties, good compatibility, and absence of toxicity and can be used either in combination with other photothermal agents or by themselves. Their magnetic properties permit them to be magnetically targeted and used as contrast agents in MRI. They have proven to effectively induce site-specific cell death in both in vivo and in vitro treatment with few side effects. However, MNPs still have some disadvantages such as a high dose of irradiation required for complete ablation of the tumor. In some cases, the irradiation exceeds the safe limit for cutaneous tissues. Further studies with MNPs must address the preparation of platforms with high thermal efficiency and total absence of side harmful effects.

\section{LIMITATIONS OF USING MAGNETIC NANOPARTICLES}

MNPs show great potential in theranostic applications and are an auspicious research field with huge and increasing interest. Representative examples of results in the diagnostic and therapeutic field have also been summarized. But some challenges remain to be overcome. The tendency to aggregate or the poor magnetization is one of the biggest challenges of the MNPs, used in biomedical applications. Chemical modification, size, shape, and assembly of MNPs strongly affect the properties of MNPs for biomedical applications. The choice of the appropriate synthesis technique is among the most essential aspects because it strongly affects the characteristics and functionality of MNPs. Therefore, it is vital to develop proper synthetic protocols with precise control of the size and shape of the different MNPs, as well as their biofunctionalization. Different approaches have permitted the synthesis of nanoparticles with wide variations among different research protocols; therefore a direct comparison of the results obtained cannot be done. Interdisciplinary approaches are needed for refinement/standardization 
of protocols of synthesis and functionalization before in vivo testing, targeting to produce nanoparticles with suitable stability, size-control, biocompatibility, and bioavailability. This should ensure that experimentations accomplished in a laboratory can more clearly match the estimated conditions that would be faced in vivo, letting proper steps to applications. Nevertheless, iron oxides are relatively less toxic as compared to other semiconductor nanomaterials or transition metals, still, some issues regarding toxicity, low biocompatibility, insufficient magnetic strength, and low drug loading capacity limit clinical translation of MNPs. 198 These issues need to be resolved soon through clear and well-established procedures for great impact on cancer diagnosis and treatment capabilities. The long-term stability of the functionalized MNPs is important before they can be widely introduced for their clinical use. Mathematical modeling of smart complex systems can also be done to understand complex interactions in the final analysis so that a given biomedical approach can be successfully used. ${ }^{199}$ The future applications and challenges in using MNPs pass through the conclusive interpretation of the toxicological features at nanoscale for both in vivo and in vitro trials. ${ }^{200}$

After several positive results in animals despite technical difficulties, few studies of magnetic drug delivery are presently undertaking initial human trials. However, it will take time before they are clinically available because they did not receive an active distribution and wide development. $^{201,202}$ Despite the promising and effective outcomes of preclinical trials of magnetic hyperthermia, there are numerous complications in this area. These comprise of establishing optimum limits of magnetic field strength and frequency, with their association with the period of treatment. The presence of specific ligands on magnetic nanoparticles improves the accumulation of MNPs in tumor cells and already proven to be effective in animal trials, but such nanoparticles remains inaccessible to humans. ${ }^{13}$ The homogenous distribution of MNPs and therapeutic temperature, and minimization of peritumoural tissue damage, need to be addressed, before hyperthermia treatment can progress to clinic. ${ }^{15}$ Tissue engineering is highly fascinating areas, in which robust research efforts have been devoted highlighting novel diagnosis and therapeutic strategies. The application of MNPs in tissue engineering has attained preliminary realization, though the interaction mechanism between MNPs, magnetic fields, and osteoblasts is not yet clear. The mechanism and principle of action need to be further clarified in the future. ${ }^{203}$ In conclusion, concerning the usage of MNPs, most of clinical trials conducted have focused on MRI so far, so that clinical valuation of other applications is probable in the near future. ${ }^{204}$ 
When magnetic nanoparticles present are used alone for PTT one of the limitations is their low molar absorption coefficient in the near infrared region. This can be fixed by controlled clustering of the nanoparticles by combining them with photothermal agents to form nano-hybrid nanocomposites thus the absorption of the indicated radiation is higher and the conversion of energy in heat is more efficient than in individual nanoparticles. ${ }^{191}$ MNPs can be designed as a therapeutic system, in which the heat generated by magnetic nanoparticles after irradiation with infrared light can release a drug attached to the nanoparticles in a controlled manner which also seems to be a promising tool of chemo-phototherapy. For such purpose, large and industrialscale production of appropriate MNPs is an important challenge to be overcome. A lot of research for the application of magnetic nanoparticles for biosensing still has to be done. The functionalization of the magnetic nanoparticles to apply in blood purification have to be developed in such a way that remove a wide range pathogens and additionally remain inert to any other cell or molecular entities in blood. Even though the progress of using magnetic nanoparticles for biosensing in possible clinical applications is visible, risk considerations may not be left out. They need to be safe for handling and treatment. ${ }^{205}$

\section{SUMMARY AND FUTURE PRESPECTIVES}

This review summarizes the importance of nanotechnology in developing inventive and advanced approaches for treatment and diagnosis with the effective nanosystems conception. Theranostics signifies a vital innovation in the progress of cancer diagnostics and therapeutics. This study shortly recaps magnetic nanoparticle's importance, synthesis, and stabilization through surface modification. In this review, we have delivered a summary on stateof-the-art of magnetic nanoparticles meant for in vivo and also in vitro experimentations used in theranostic applications. In biomedical application MNPs, particularly SPIONs have emerged with a range of formulations for bioimaging and therapeutic applications. The research work on the fabrication of tremendously active nanovectors for in vivo and in vitro investigations carried out practically exclusively with magnetic nanoparticles is reasonable and understandable and has been established and explored since many years. Their stability, ability to targeting dispersibility, and biocompatibility can be enhanced by functionalization of surface with biocompatible polymers, to modify the particle chemical functionality and physical properties. Reliable protocols are available for the surface modification and functionalization for reduced 
cytotoxicity. Nevertheless, significant efforts have been conducted for preliminary clinical trials of hyperthermia manipulating magnetic colloidal mediators. Substantial research was done for surface stabilization of magnetic nanoparticles to prove their safety, bio distribution, stability, and toxicity by incorporating suitable natural or synthetic polymer.

In conclusion, our review describes the potential applications of the nanometer-size platforms that accomplish numerous tasks instantaneously. Noticeably an extraordinary level of obstacle in magnetic nanoparticle is to attain multimodal imaging or collective theranostic abilities. MNPs are routinely used as a contrast agent with association with drug delivery that allows researchers to envision great advances. The foremost development to improve and advance nanomaterials is to fabricate them multi-functional controlled by signals externally applied or by local surroundings turning them into smart and functional nanomaterials. MNPs are engineered as essential constituents of assays, therapeutics, imaging agents, multifunctional devices and sensors. To attain extensive recognition in medical applications, more scientific and technical research is required to apprehend the physical and chemical characteristics of MNPs. MNPs have not being completely grasped their optimum well-being and safety for in vivo applications for routine qualitative and quantitative theranostic tools yet. Their limitations can be overpowered through the upgrading of magnetic targeted carriers by continuous research and pre-clinical experimentation. A recognizable cooperative conciliation between biochemistry, materials science, nanotechnology, and medical investigation is essential to accomplish the required goals and objectives completely.

Hence, magnetic nanoparticle and biomedical approaches signify a broad and strong interdisciplinary research and application field. Significant advances have been accomplished in the improvement of suitable MNPs and their functionalization. Remarkable proofs-of-concept in a large variety of theranostic areas have confirmed the full potential of this research area, demonstrating an important evolution in the entire biomedical field. The future is hopeful as well as thought-provoking and novel research concepts are needed to be worked out to avoid its limitations for theranostic applications. MNPs own a strong candidature for non-aqueous/aqeous phase solubility and are promising for in vitro and in vivo applications. To achieve this target numerous factorsplay a key-role including suitable precursors, $\mathrm{pH}$ of the medium, coating agents and solvents for the synthesis of MNPs. Aqueous phase solubility can be expressively enhanced by using water-soluble surface functionalization agents by thermal decomposition, co- 
precipitation, and microwave and high-temperature methods.

The scientific information collected in this review unlocks innovative perceptions into the role of MNPs to develop nanohybrid composites to escalate their efficacy especially in the area of disease diagnosis, early detection, cellular and deep tissue imaging, drug/gene delivery as well as modern-day theranostics. The present importance of molecular medicine is to advance new unique tools for early-stage disease diagnosis and more of point-of-care diagnostics. Integration of MNPs especially IONPs, encompass the fabrication of theranostic platform that syndicates therapeutics with diagnostics. Such efforts would mainly make the diagnosis processes simple, speedy and less invasive. Personalized medicine is also attaining consideration and it is predictable that the integration of nanotechnology could effect in overarching conclusions. In near-term, multifunctional MNPS would be an eye-catching material for theranostic applications and may change the usual business model of pharmaceutical industries. We consider that these progressive reports will direct the academic researchers and industries to precise the contemporary translational stage for the further applications using these multifunctionalized MNPs.

\section{AUTHOR INFORMATION}

*Corresponding Authors

Abdelhamid Elaissari- Univ Lyon, Université Claude Bernard Lyon-1, CNRS, ISA-UMR 5280, F-69622 Lyon, France; Email: abdelhamid.elaissari@univ-lyon1.fr

Nasir M. Ahmad- Polymer Research Lab, School of Chemical and Materials Engineering (SCME), National University of Sciences and Technology (NUST), H-12 Sector, Islamabad44000, Pakistan; Email: nasir.ahmad@ scme.nust.edu.pk

\section{Authors}

Sumera Khizar- Polymer Research Lab, School of Chemical and Materials Engineering (SCME), National University of Sciences and Technology (NUST), H-12 Sector, Islamabad44000, Pakistan

Nadia Zine- Univ Lyon, Université Claude Bernard Lyon-1, CNRS, ISA-UMR 5280, F-69622 Lyon, France

Nicole Jaffrezic- Renault- Univ Lyon, Université Claude Bernard Lyon-1, CNRS, ISA-UMR 5280, F-69622 Lyon, France 
Abdelhamid Errachid-el-salhi - Univ Lyon, Université Claude Bernard Lyon-1, CNRS, ISAUMR 5280, F-69622 Lyon, France

\section{Author Contributions}

The manuscript was written by Sumera Khizar. Abdelhamid Elaissari edited and finalized the manuscript. All authors have approved the final version of the manuscript.

\section{Notes}

The authors declare no competing financial interest.

\section{ACKNOWLEDGMENTS}

Sumera Khizar acknowledges the financial support of HEC for the scholarship and IRSIP program [grant No. 9046] for visit to LAGEPP-UMR, University Claude Bernard Lyon-1, Lyon, France.

\section{REFERENCES}

1. Sagayaraj, R.; Jegadheeswari, M.; Aravazhi, S.; Chandrasekaran, G.; Dhanalakshmi, A., Structural, Spectroscopic and Magnetic Study of Nanocrystalline Terbium-Nickel Ferrite by Oxalate Co-Precipitation Method. Chemistry Africa 2020, 1-9.

2. Gul, S.; Khan, S. B.; Rehman, I. U.; Khan, M. A.; Khan, M. I., A Comprehensive Review of Magnetic Nanomaterials Modern Day Theranostics. Frontiers in Materials 2019, 6, 1-15.

3. Lima-Tenorio, M. K.; Pineda, E. A.; Ahmad, N. M.; Fessi, H.; Elaissari, A., Magnetic nanoparticles: In vivo cancer diagnosis and therapy. International journal of pharmaceutics 2015, 493 (1-2), 313-27.

4. Wang, J.; Cui, H., Nanostructure-Based Theranostic Systems. Theranostics 2016, 6 (9), 1274-6.

5. Ferreira, M.; Sousa, J.; Pais, A.; Vitorino, C., The Role of Magnetic Nanoparticles in Cancer Nanotheranostics. Materials 2020, 13 (2), 266.

6. Tarhini, M.; Vega-Chacón, J.; Jafelicci, M.; Zine, N.; Errachid, A.; Fessi, H.; Elaissari, A., Structured Magnetic Core/Silica Internal Shell Layer and Protein Out Layer Shell (BSA@SiO2@SME): Preparation and Characterization. Chemistry Africa 2019, 3 (1), 127-134.

7. Kolhatkar, A. G.; Jamison, A. C.; Litvinov, D.; Willson, R. C.; Lee, T. R., Tuning the magnetic properties of nanoparticles. International journal of molecular sciences 2013, 14 (8), 15977-6009.

8. Biehl, P.; Von der Luhe, M.; Dutz, S.; Schacher, F. H., Synthesis, Characterization, and Applications of Magnetic Nanoparticles Featuring Polyzwitterionic Coatings. Polymers (Basel) 2018, 10 (1), 91.

9. Leem, G.; Sarangi, S.; Zhang, S.; Rusakova, I.; Brazdeikis, A.; Litvinov, D.; Lee, T. R., SurfactantControlled Size and Shape Evolution of Magnetic Nanoparticles. Crystal Growth \& Design 2009, 9 (1), 3234.

10. Xiao, Y.; Du, J., Superparamagnetic nanoparticles for biomedical applications. Journal of materials chemistry. B 2020, 8 (3), 354-367.

11. Israel, L. L.; Galstyan, A.; Holler, E.; Ljubimova, J. Y., Magnetic iron oxide nanoparticles for imaging, targeting and treatment of primary and metastatic tumors of the brain. Journal of controlled release : official journal of the Controlled Release Society 2020, 320, 45-62.

12. Bucharskaya, A. B., The Magnetite Nanoparticles in Theranostic Applications. Journal of Nanomedicine Research 2017, 5 (3), 00119. 
13. Shabatina, T. I.; Vernaya, O. I.; Shabatin, V. P.; Melnikov, M. Y., Magnetic Nanoparticles for Biomedical Purposes: Modern Trends and Prospects. Magnetochemistry 2020, 6 (3), 30.

14. Cortajarena, A. L.; Ortega, D.; Ocampo, S. M.; Gonzalez-Garcia, A.; Couleaud, P.; Miranda, R.; Belda-Iniesta, C.; Ayuso-Sacido, A., Engineering Iron Oxide Nanoparticles for Clinical Settings. Nanobiomedicine 2014, 1, 2.

15. Chang, D.; Lim, M.; Goos, J.; Qiao, R.; Ng, Y. Y.; Mansfeld, F. M.; Jackson, M.; Davis, T. P.; Kavallaris, M., Biologically Targeted Magnetic Hyperthermia: Potential and Limitations. Frontiers in pharmacology 2018, 9, 831.

16. Ramanathan, S.; Archunan, G.; Sivakumar, M.; Tamil Selvan, S.; Fred, A. L.; Kumar, S.; Gulyas, B.; Padmanabhan, P., Theranostic applications of nanoparticles in neurodegenerative disorders. International journal of nanomedicine 2018, 13, 5561-5576.

17. Yang, W.; Veroniaina, H.; Qi, X.; Chen, P.; Li, F.; Ke, P. C., Soft and Condensed Nanoparticles and Nanoformulations for Cancer Drug Delivery and Repurpose. Advanced Therapeutics 2019, 3 (1), 1900102.

18. Ladj, R.; Bitar, A.; Eissa, M.; Mugnier, Y.; Le Dantec, R.; Fessi, H.; Elaissari, A., Individual inorganic nanoparticles: preparation, functionalization and in vitro biomedical diagnostic applications. Journal of materials chemistry. B 2013, 1 (10), 1381-1396.

19. Kim, B. S.; Lee, T. R., The Development of Smart, Multi-Responsive Core@Shell Composite Nanoparticles. Nanoparticles Technology 2015, 1-22.

20. Mohapatra, A.; Uthaman, S.; Park, I. K., External and Internal Stimuli-Responsive Metallic Nanotherapeutics for Enhanced Anticancer Therapy. Frontiers in molecular biosciences 2020, 7, 597634.

21. Yaqoob, S. B.; Adnan, R.; Rameez Khan, R. M.; Rashid, M., Gold, Silver, and Palladium Nanoparticles: A Chemical Tool for Biomedical Applications. Frontiers in chemistry 2020, 8, 376.

22. Zhang, D.; Ma, X. L.; Gu, Y.; Huang, H.; Zhang, G. W., Green Synthesis of Metallic Nanoparticles and Their Potential Applications to Treat Cancer. Frontiers in chemistry 2020, 8, 799.

23. Alirezaie Alavijeh, A.; Barati, M.; Barati, M.; Abbasi Dehkordi, H., The Potential of Magnetic Nanoparticles for Diagnosis and Treatment of Cancer Based on Body Magnetic Field and Organ-on-theChip. Advanced pharmaceutical bulletin 2019, 9 (3), 360-373.

24. Kim, J. H.; Bryan, W. W.; Lee, T. R., Preparation, characterization, and optical properties of gold, silver, and gold-silver alloy nanoshells having silica cores. Langmuir : the ACS journal of surfaces and colloids 2008, 24 (19), 11147-52.

25. Balakrishnan, S.; Mukherjee, S.; Das, S.; Bhat, F. A.; Raja Singh, P.; Patra, C. R.; Arunakaran, J., Gold nanoparticles-conjugated quercetin induces apoptosis via inhibition of EGFR/PI3K/Akt-mediated pathway in breast cancer cell lines (MCF-7 and MDA-MB-231). Cell biochemistry and function 2017, 35 (4), 217-231.

26. Mukherjee, S.; Chowdhury, D.; Kotcherlakota, R.; Patra, S.; B, V.; Bhadra, M. P.; Sreedhar, B.; Patra, C. R., Potential theranostics application of bio-synthesized silver nanoparticles (4-in-1 system). Theranostics 2014, 4 (3), 316-35.

27. Rampado, R.; Crotti, S.; Caliceti, P.; Pucciarelli, S.; Agostini, M., Nanovectors Design for Theranostic Applications in Colorectal Cancer. Journal of oncology 2019, 2019, 2740923.

28. Sarcan, E. T.; Silindir-Gunay, M.; Ozer, A. Y., Theranostic polymeric nanoparticles for NIR imaging and photodynamic therapy. International journal of pharmaceutics 2018, 551 (1-2), 329-338.

29. Saqib, M.; Ali Bhatti, A. S.; Ahmad, N. M.; Ahmed, N.; Shahnaz, G.; Lebaz, N.; Elaissari, A., Amphotericin B Loaded Polymeric Nanoparticles for Treatment of Leishmania Infections. Nanomaterials 2020, $10(6), 1152$.

30. Iqbal, M.; Fessi, H.; Elaissari, A., Elaboration of Submicron Particles for Biomedical Imaging and Drug Delivery: Specific Review. Journal of Colloid Science and Biotechnology 2016, 5 (1), 16-31. 
31. Kenny, G. D.; Kamaly, N.; Kalber, T. L.; Brody, L. P.; Sahuri, M.; Shamsaei, E.; Miller, A. D.; Bell, J. D., Novel multifunctional nanoparticle mediates siRNA tumour delivery, visualisation and therapeutic tumour reduction in vivo. Journal of controlled release : official journal of the Controlled Release Society 2011, 149 (2), 111-6.

32. Zottel, A.; Videtic Paska, A.; Jovcevska, I., Nanotechnology Meets Oncology: Nanomaterials in Brain Cancer Research, Diagnosis and Therapy. Materials 2019, 12 (10), 1588.

33. Onoshima, D.; Yukawa, H.; Baba, Y., Multifunctional quantum dots-based cancer diagnostics and stem cell therapeutics for regenerative medicine. Advanced drug delivery reviews 2015, 95, 2-14.

34. Singh, R. P.; Sharma, G.; Sonali; Singh, S.; Kumar, M.; Pandey, B. L.; Koch, B.; Muthu, M. S., Vitamin E TPGS conjugated carbon nanotubes improved efficacy of docetaxel with safety for lung cancer treatment. Colloids and surfaces. B, Biointerfaces 2016, 141, 429-442.

35. d'Angelo, M.; Castelli, V.; Benedetti, E.; Antonosante, A.; Catanesi, M.; Dominguez-Benot, R.; Pitari, G.; Ippoliti, R.; Cimini, A., Theranostic Nanomedicine for Malignant Gliomas. Frontiers in bioengineering and biotechnology 2019, 7, 325.

36. Mukherjee, A.; Paul, M.; Mukherjee, S., Recent Progress in the Theranostics Application of Nanomedicine in Lung Cancer. Cancers 2019, 11 (5), 597.

37. Bolanos, K.; Kogan, M. J.; Araya, E., Capping gold nanoparticles with albumin to improve their biomedical properties. International journal of nanomedicine 2019, 14, 6387-6406.

38. Kim, J.-H.; Randall Lee, T., Discrete thermally responsive hydrogel-coated gold nanoparticles for use as drug-delivery vehicles. Drug Development Research 2006, 67 (1), 61-69.

39. Nakamura, S.; Sato, M.; Sato, Y.; Ando, N.; Takayama, T.; Fujita, M.; Ishihara, M., Synthesis and Application of Silver Nanoparticles (Ag NPs) for the Prevention of Infection in Healthcare Workers. International journal of molecular sciences 2019, 20 (15), 3620.

40. Modugno, G.; Menard-Moyon, C.; Prato, M.; Bianco, A., Carbon nanomaterials combined with metal nanoparticles for theranostic applications. British journal of pharmacology 2015, 172 (4), 975-91.

41. Iturrioz-Rodriguez, N.; Correa-Duarte, M. A.; Fanarraga, M. L., Controlled drug delivery systems for cancer based on mesoporous silica nanoparticles. International journal of nanomedicine 2019, 14, 3389-3401.

42. Ranucci, E.; Manfredi, A., Polyamidoamines: Versatile Bioactive Polymers with Potential for Biotechnological Applications. Chemistry Africa 2019, 2 (2), 167-193.

43. Ocsoy, I.; Tasdemir, D.; Mazicioglu, S.; Celik, C.; Katı, A.; Ulgen, F., Biomolecules incorporated metallic nanoparticles synthesis and their biomedical applications. Materials Letters 2018, 212, 45-50.

44. Chen, Y. T.; Kolhatkar, A. G.; Zenasni, O.; Xu, S.; Lee, T. R., Biosensing Using Magnetic Particle Detection Techniques. Sensors 2017, 17 (10), 2300.

45. Ali, A.; Zafar, H.; Zia, M.; UI Haq, I.; Phull, A. R.; Ali, J. S.; Hussain, A., Synthesis, characterization, applications, and challenges of iron oxide nanoparticles. Nanotechnology, science and applications 2016, 9, 49-67.

46. Verma, J.; Lal, S.; Van Noorden, C. J., Nanoparticles for hyperthermic therapy: synthesis strategies and applications in glioblastoma. International journal of nanomedicine 2014, 9, 2863-77.

47. Chen, Q.; Zhang, Z. J., Size-dependent superparamagnetic properties of MgFe2O4 spinel ferrite nanocrystallites. Applied Physics Letters 1998, 73 (21), 3156-3158.

48. Silva, V. A. J.; Andrade, P. L.; Silva, M. P. C.; Bustamante D, A.; De Los Santos Valladares, L.; Albino Aguiar, J., Synthesis and characterization of Fe3O4 nanoparticles coated with fucan polysaccharides. Journal of Magnetism and Magnetic Materials 2013, 343, 138-143.

49. Poddar, P.; Wilson, J. L.; Srikanth, H.; Morrison, S. A.; Carpenter, E. E., Magnetic properties of conducting polymer doped with manganese-zinc ferrite nanoparticles. Nanotechnology 2004, 15 (10), S570-S574. 
50. Das, N.; Majumdar, R.; Sen, A.; Maiti, H. S., Nanosized bismuth ferrite powder prepared through sonochemical and microemulsion techniques. Materials Letters 2007, 61 (10), 2100-2104.

51. Tago, T.; Hatsuta, T.; Miyajima, K.; Kishida, M.; Tashiro, S.; Wakabayashi, K., Novel Synthesis of Silica-Coated Ferrite Nanoparticles Prepared Using Water-in-Oil Microemulsion. Journal of the American Ceramic Society 2002, 85 (9), 2188-2194.

52. Suh, W. H.; Suslick, K. S., Magnetic and porous nanospheres from ultrasonic spray pyrolysis. Journal of the American Chemical Society 2005, 127 (34), 12007-10.

53. Itoh, H.; Sugimoto, T., Systematic control of size, shape, structure, and magnetic properties of uniform magnetite and maghemite particles. Journal of Colloid and Interface Science 2003, 265 (2), 283-295.

54. Figueroa, S.; Desimoni, J.; Rivas, P. C.; Cervera, M. M.; Caracoche, M. C.; de Sanctis, O., Hyperfine Study on Sol-Gel Derived-Hematite Doped Zirconia. Chemistry of Materials 2005, 17 (13), 3486-3491.

55. Xing, L.; Ten Brink, G. H.; Chen, B.; Schmidt, F. P.; Haberfehlner, G.; Hofer, F.; Kooi, B. J.; Palasantzas, G., Synthesis and morphology of iron-iron oxide core-shell nanoparticles produced by high pressure gas condensation. Nanotechnology 2016, 27 (21), 215703.

56. Madamsetty, V. S.; Mukherjee, A.; Mukherjee, S., Recent Trends of the Bio-Inspired Nanoparticles in Cancer Theranostics. Frontiers in pharmacology 2019, 10, 1264.

57. Katz, Synthesis, Properties and Applications of Magnetic Nanoparticles and Nanowires-A Brief Introduction. Magnetochemistry 2019, 5 (4), 61.

58. Baranwal, A.; Mahato, K.; Srivastava, A.; Maurya, P. K.; Chandra, P., Phytofabricated metallic nanoparticles and their clinical applications. RSC Advances 2016, 6 (107), 105996-106010.

59. Sandler, S. E.; Fellows, B.; Mefford, O. T., Best Practices for Characterization of Magnetic Nanoparticles for Biomedical Applications. Analytical chemistry 2019, 91 (22), 14159-14169.

60. Nahar, Y.; Rahman, M. A.; Hossain, M. K.; Sharafat, M. K.; Karim, M. R.; Elaissari, A.; Ochiai, B.; Ahmad, H.; Rahman, M. M., A facile one-pot synthesis of poly(acrylic acid)-functionalized magnetic iron oxide nanoparticles for suppressing reactive oxygen species generation and adsorption of biocatalyst. Materials Research Express 2019, 7 (1), 016102.

61. Kostevšek, N., A Review on the Optimal Design of Magnetic Nanoparticle-Based T2 MRI Contrast Agents. Magnetochemistry 2020, 6 (1), 11.

62. Socoliuc, V.; Peddis, D.; Petrenko, V. I.; Avdeev, M. V.; Susan-Resiga, D.; Szabó, T.; Turcu, R.; Tombácz, E.; Vékás, L., Magnetic Nanoparticle Systems for Nanomedicine-A Materials Science Perspective. Magnetochemistry 2020, 6 (1), 2.

63. Khan, I.; Saeed, K.; Khan, I., Nanoparticles: Properties, applications and toxicities. Arabian Journal of Chemistry 2019, 12 (7), 908-931.

64. Kim, B. S.; Chen, Y. T.; Srinoi, P.; Marquez, M. D.; Lee, T. R., Hydrogel-Encapsulated Mesoporous Silica-Coated Gold Nanoshells for Smart Drug Delivery. International journal of molecular sciences 2019, 20 (14), 3422.

65. Pakdel, S.; Akhlaghinia, B.; Mohammadinezhad, A., Fe304@Boehmite-NH2-Coll NPs: An Environment Friendly Nanocatalyst for Solvent Free Synthesis of Coumarin Derivatives Through Pechmann Condensation Reaction. Chemistry Africa 2019, 2 (3), 367-376.

66. Ladj, R.; Bitar, A.; Eissa, M. M.; Fessi, H.; Mugnier, Y.; Le Dantec, R.; Elaissari, A., Polymer encapsulation of inorganic nanoparticles for biomedical applications. International journal of pharmaceutics 2013, 458 (1), 230-41.

67. Wu, W.; Wu, Z.; Yu, T.; Jiang, C.; Kim, W. S., Recent progress on magnetic iron oxide nanoparticles: synthesis, surface functional strategies and biomedical applications. Science and technology of advanced materials 2015, 16 (2), 023501. 
68. Rittikulsittichai, S.; Kolhatkar, A. G.; Sarangi, S.; Vorontsova, M. A.; Vekilov, P. G.; Brazdeikis, A.; Randall Lee, T., Multi-responsive hybrid particles: thermo-, $\mathrm{pH}-$, photo-, and magneto-responsive magnetic hydrogel cores with gold nanorod optical triggers. Nanoscale 2016, 8 (23), 11851-61.

69. Veiseh, O.; Gunn, J. W.; Zhang, M., Design and fabrication of magnetic nanoparticles for targeted drug delivery and imaging. Advanced drug delivery reviews 2010, 62 (3), 284-304.

70. Nawaz, M.; Sliman, Y.; Ercan, I.; Lima-Tenório, M. K.; Tenório-Neto, E. T.; Kaewsaneha, C.; Elaissari, A., Stimuli Responsive Polymeric Nanocarriers for Drug Delivery Applications. 2019; Vol. 2, p 3785.

71. Shakiba, A.; Zenasni, O.; D. Marquez, M.; Randall Lee, T., Advanced drug delivery via selfassembled monolayer-coated nanoparticles. AIMS Bioengineering 2017, 4 (2), 275-299.

72. Guo, M.; Yan, Y.; Zhang, H.; Yan, H.; Cao, Y.; Liu, K.; Wan, S.; Huang, J.; Yue, W., Magnetic and $\mathrm{pH}$-responsive nanocarriers with multilayer core-shell architecture for anticancer drug delivery. Journal of Materials Chemistry 2008, 18 (42), 5104.

73. Yen, S. K.; Padmanabhan, P.; Selvan, S. T., Multifunctional iron oxide nanoparticles for diagnostics, therapy and macromolecule delivery. Theranostics 2013, 3 (12), 986-1003.

74. Tanasa, E.; Zaharia, C.; Radu, I. C.; Surdu, V. A.; Vasile, B. S.; Damian, C. M.; Andronescu, E., Novel Nanocomposites Based on Functionalized Magnetic Nanoparticles and Polyacrylamide: Preparation and Complex Characterization. Nanomaterials 2019, 9 (10), 1384.

75. Zhao, S.; Yu, X.; Qian, Y.; Chen, W.; Shen, J., Multifunctional magnetic iron oxide nanoparticles: an advanced platform for cancer theranostics. Theranostics 2020, 10 (14), 6278-6309.

76. Taddei, C.; Sansone, L.; Ausanio, G.; lannotti, V.; Pepe, G. P.; Giordano, M.; Serra, C. A., Fabrication of polystyrene-encapsulated magnetic iron oxide nanoparticles via batch and microfluidicassisted production. Colloid and polymer science 2019, 297 (6), 861-870.

77. Cordeiro, A. P.; Feuser, P. E.; Araújo, P. H. H.; Sayer, C., Encapsulation of Magnetic Nanoparticles and Copaíba Oil in Poly(methyl methacrylate) Nanoparticles via Miniemulsion Polymerization for Biomedical Application. Macromolecular Symposia 2020, 394 (1), 2000112.

78. Kojima, R.; Hidaka, S.; Taira, M.; Kohri, M.; Taniguchi, T.; Kishikawa, K.; Karatsu, T.; Okabe, E.; Kondo, F., Preparation of liquid crystal nanocapsules by polymerization of oil-in-water emulsion monomer droplets. J Colloid Interface Sci 2020, 563, 122-130.

79. Sanchez, A.; Mejia, S. P.; Orozco, J., Recent Advances in Polymeric Nanoparticle-Encapsulated Drugs against Intracellular Infections. Molecules 2020, 25 (16), 11.

80. Nayeem, J.; Al-Bari, M. A. A.; Mahiuddin, M.; Rahman, M. A.; Mefford, O. T.; Ahmad, H.; Rahman, M. M., Silica coating of iron oxide magnetic nanoparticles by reverse microemulsion method and their functionalization with cationic polymer P(NIPAm-co-AMPTMA) for antibacterial vancomycin immobilization. Colloids and Surfaces A: Physicochemical and Engineering Aspects 2021, 611, 125857.

81. Lu, Y.; Lin, J.; Wang, L.; Zhang, L.; Cai, C., Self-Assembly of Copolymer Micelles: Higher-Level Assembly for Constructing Hierarchical Structure. Chemical reviews 2020, 120 (9), 4111-4140.

82. Tufani, A.; Qureshi, A.; Niazi, J. H., Iron oxide nanoparticles based magnetic luminescent quantum dots (MQDs) synthesis and biomedical/biological applications: A review. Materials science \& engineering. C, Materials for biological applications 2021, 118, 111545.

83. Iqbal, M.; Zafar, N.; Fessi, H.; Elaissari, A., Double emulsion solvent evaporation techniques used for drug encapsulation. International journal of pharmaceutics 2015, 496 (2), 173-90.

84. Badri, W.; Miladi, K.; Nazari, Q. A.; Fessi, H.; Elaissari, A., Effect of process and formulation parameters on polycaprolactone nanoparticles prepared by solvent displacement. Colloids and Surfaces A: Physicochemical and Engineering Aspects 2017, 516, 238-244.

85. Ahmed, N.; Michelin-Jamois, M.; Fessi, H.; Elaissari, A., Modified double emulsion process as a new route to prepare submicron biodegradable magnetic/polycaprolactone particles for in vivo theranostics. Soft Matter 2012, 8 (8), 2554. 
86. Ahmed, N.; Fessi, H.; Elaissari, A., Theranostic applications of nanoparticles in cancer. Drug discovery today 2012, 17 (17-18), 928-34.

87. Khizar, S.; Ahmad, N. M.; Ahmed, N.; Manzoor, S.; Elaissari, A., Encapsulation of doxorubicin in magnetic-polymer hybrid colloidal particles of Eudragit E100 and their hyperthermia and drug release studies. Polymers for Advanced Technologies 2020, 31 (8), 1732-1743.

88. Stephen, Z. R.; Kievit, F. M.; Zhang, M., Magnetite nanoparticles for medical MR imaging. Materials Today 2011, 14 (7-8), 330-338.

89. Pankhurst, Q. A.; Connolly, J.; Jones, S. K.; Dobson, J., Applications of magnetic nanoparticles in biomedicine. Journal of Physics D: Applied Physics 2003, 36 (13), R167-R181.

90. Estelrich, J.; Sanchez-Martin, M. J.; Busquets, M. A., Nanoparticles in magnetic resonance imaging: from simple to dual contrast agents. International journal of nanomedicine 2015, 10, 1727-41.

91. Johannsen, M.; Gneveckow, U.; Thiesen, B.; Taymoorian, K.; Cho, C. H.; Waldofner, N.; Scholz, R.; Jordan, A.; Loening, S. A.; Wust, P., Thermotherapy of prostate cancer using magnetic nanoparticles: feasibility, imaging, and three-dimensional temperature distribution. European urology 2007, 52 (6), 1653-61.

92. Ito, A.; Tanaka, K.; Honda, H.; Abe, S.; Yamaguchi, H.; Kobayashi, T., Complete regression of mouse mammary carcinoma with a size greater than $15 \mathrm{~mm}$ by frequent repeated hyperthermia using magnetite nanoparticles. Journal of Bioscience and Bioengineering 2003, 96 (4), 364-369.

93. Ito, A.; Shinkai, M.; Honda, H.; Kobayashi, T., Medical application of functionalized magnetic nanoparticles. J Biosci Bioeng 2005, 100 (1), 1-11.

94. Veyret, R.; Delair, T.; Elaissari, A., Preparation and biomedical application of layer-by-layer encapsulated oil in water magnetic emulsion. Journal of Magnetism and Magnetic Materials 2005, 293 (1), 171-176.

95. Vega-Chacón, J.; Tarhini, M.; Lebaz, N.; Jafelicci, M.; Zine, N.; Errachid, A.; Elaissari, A., ProteinSilica Hybrid Submicron Particles: Preparation and Characterization. Chemistry Africa 2020, 1-10.

96. Bamburowicz-Klimkowska, M.; Poplawska, M.; Grudzinski, I. P., Nanocomposites as biomolecules delivery agents in nanomedicine. Journal of nanobiotechnology 2019, 17 (1), 48.

97. Rittikulsittichai, S.; Singhana, B.; Bryan, W. W.; Sarangi, S.; Jamison, A. C.; Brazdeikis, A.; Lee, T. R., Preparation, characterization, and utilization of multi-functional magnetic-fluorescent composites for bio-imaging and magnetic hyperthermia therapy. RSC Advances 2013, 3 (21), 7838.

98. German, S. V.; Navolokin, N. A.; Kuznetsova, N. R.; Zuev, V. V.; Inozemtseva, O. A.; Anis'kov, A. A.; Volkova, E. K.; Bucharskaya, A. B.; Maslyakova, G. N.; Fakhrullin, R. F.; Terentyuk, G. S.; Vodovozova, E. L.; Gorin, D. A., Liposomes loaded with hydrophilic magnetite nanoparticles: Preparation and application as contrast agents for magnetic resonance imaging. Colloids and surfaces. B, Biointerfaces 2015, 135, 109-115.

99. Song, X.; Zhang, Y.; Sun, H.; Dong, P.; Zhang, Z.; Liu, J., In Vitro Safety Evaluation and In Vivo Imaging Studies of Superparamagnetic Iron Oxide Nanoparticles through Biomimetic Modification. Journal of Nanomaterials 2019, 2019, 1-9.

100. Moradi Khaniabadi, P.; Shahbazi-Gahrouei, D.; Malik Shah Abdul Majid, A.; Suhaimi Jaafar, M.; Moradi Khaniabadi, B.; Shahbazi-Gahrouei, S., In vitro Study of SPIONs-C595 as Molecular Imaging Probe for Specific Breast Cancer (MCF-7) Cells Detection. Iranian biomedical journal 2017, 21 (6), 360-8.

101. Lazaro-Carrillo, A.; Filice, M.; Guillen, M. J.; Amaro, R.; Vinambres, M.; Tabero, A.; Paredes, K. O.; Villanueva, A.; Calvo, P.; Del Puerto Morales, M.; Marciello, M., Tailor-made PEG coated iron oxide nanoparticles as contrast agents for long lasting magnetic resonance molecular imaging of solid cancers. Materials science \& engineering. C, Materials for biological applications 2020, 107, 110262.

102. Ahmed, N.; Jaafar-Maalej, C.; Eissa, M. M.; Fessi, H.; Elaissari, A., New oil-in-water magnetic emulsion as contrast agent for in vivo magnetic resonance imaging (MRI). Journal of biomedical nanotechnology 2013, 9 (9), 1579-85. 
103. Ju, K. Y.; Lee, J. W.; Im, G. H.; Lee, S.; Pyo, J.; Park, S. B.; Lee, J. H.; Lee, J. K., Bio-inspired, melanin-like nanoparticles as a highly efficient contrast agent for T1-weighted magnetic resonance imaging. Biomacromolecules 2013, 14 (10), 3491-7.

104. Iqbal, M. Z.; Ma, X.; Chen, T.; Zhang, L.; Ren, W.; Xiang, L.; Wu, A., Silica-coated superparamagnetic iron oxide nanoparticles (SPIONPS): a new type contrast agent of T1 magnetic resonance imaging (MRI). Journal of materials chemistry. B 2015, 3 (26), 5172-5181.

105. Tao, C.; Zheng, Q.; An, L.; He, M.; Lin, J.; Tian, Q.; Yang, S., T(1)-Weight Magnetic Resonance Imaging Performances of Iron Oxide Nanoparticles Modified with a Natural Protein Macromolecule and an Artificial Macromolecule. Nanomaterials 2019, 9 (2), 170.

106. Pellico, J.; Ruiz-Cabello, J.; Fernandez-Barahona, I.; Gutierrez, L.; Lechuga-Vieco, A. V.; Enriquez, J. A.; Morales, M. P.; Herranz, F., One-Step Fast Synthesis of Nanoparticles for MRI: Coating Chemistry as the Key Variable Determining Positive or Negative Contrast. Langmuir : the ACS journal of surfaces and colloids 2017, 33 (39), 10239-10247.

107. Vangijzegem, T.; Stanicki, D.; Boutry, S.; Paternoster, Q.; Vander Elst, L.; Muller, R. N.; Laurent, S., VSION as high field MRI T1 contrast agent: evidence of their potential as positive contrast agent for magnetic resonance angiography. Nanotechnology 2018, 29 (26), 265103.

108. Lima-Tenorio, M. K.; Pineda, E. A. G.; Ahmad, N. M.; Agusti, G.; Manzoor, S.; Kabbaj, D.; Fessi, H.; Elaissari, A., Aminodextran polymer-functionalized reactive magnetic emulsions for potential theranostic applications. Colloids and surfaces. B, Biointerfaces 2016, 145, 373-381.

109. Khizar, S.; Ahmad, N. M.; Ahmed, N.; Manzoor, S.; Hamayun, M. A.; Naseer, N.; Tenorio, M. K. L.; Lebaz, N.; Elaissari, A., Aminodextran Coated CoFe2O4 Nanoparticles for Combined Magnetic Resonance Imaging and Hyperthermia. Nanomaterials 2020, 10 (11), 2182.

110. Thapa, B.; Diaz-Diestra, D.; Santiago-Medina, C.; Kumar, N.; Tu, K.; Beltran-Huarac, J.; Jadwisienczak, W. M.; Weiner, B. R.; Morell, G., T1- and T2-weighted Magnetic Resonance Dual Contrast by Single Core Truncated Cubic Iron Oxide Nanoparticles with Abrupt Cellular Internalization and Immune Evasion. ACS applied bio materials 2018, 1 (1), 79-89.

111. Xiao, S.; Yu, X.; Zhang, L.; Zhang, Y.; Fan, W.; Sun, T.; Zhou, C.; Liu, Y.; Liu, Y.; Gong, M.; Zhang, D., Synthesis Of PEG-Coated, Ultrasmall, Manganese-Doped Iron Oxide Nanoparticles With High Relaxivity For T1/T2 Dual-Contrast Magnetic Resonance Imaging. International journal of nanomedicine 2019, 14, 8499-8507.

112. Dulinska-Litewka, J.; Lazarczyk, A.; Halubiec, P.; Szafranski, O.; Karnas, K.; Karewicz, A., Superparamagnetic Iron Oxide Nanoparticles-Current and Prospective Medical Applications. Materials 2019, 12 (4), 617.

113. Vallabani, N. V. S.; Singh, S., Recent advances and future prospects of iron oxide nanoparticles in biomedicine and diagnostics. 3 Biotech 2018, 8 (6), 279.

114. Reguera, J.; Jimenez de Aberasturi, D.; Henriksen-Lacey, M.; Langer, J.; Espinosa, A.; Szczupak, B.; Wilhelm, C.; Liz-Marzan, L. M., Janus plasmonic-magnetic gold-iron oxide nanoparticles as contrast agents for multimodal imaging. Nanoscale 2017, 9 (27), 9467-9480.

115. Cai, J.; Miao, Y. Q.; Li, L.; Fan, H. M., Facile Preparation of Gold-Decorated Fe(3)O(4) Nanoparticles for CT and MR Dual-Modal Imaging. International journal of molecular sciences 2018, 19 (12), 4049.

116. Naha, P. C.; Zaki, A. A.; Hecht, E.; Chorny, M.; Chhour, P.; Blankemeyer, E.; Yates, D. M.; Witschey, W. R.; Litt, H. I.; Tsourkas, A.; Cormode, D. P., Dextran coated bismuth-iron oxide nanohybrid contrast agents for computed tomography and magnetic resonance imaging. Journal of materials chemistry. B 2014, 2 (46), 8239-8248.

117. Perlman, O.; Azhari, H., Ultrasonic computed tomography imaging of iron oxide nanoparticles. Physics in medicine and biology 2017, 62 (3), 825-842. 
118. Feng, W.; Zhou, X.; Nie, W.; Chen, L.; Qiu, K.; Zhang, Y.; He, C., Au/polypyrrole@Fe3O4 nanocomposites for MR/CT dual-modal imaging guided-photothermal therapy: an in vitro study. ACS applied materials \& interfaces 2015, 7 (7), 4354-67.

119. Das, P.; Colombo, M.; Prosperi, D., Recent advances in magnetic fluid hyperthermia for cancer therapy. Colloids and surfaces. B, Biointerfaces 2019, 174, 42-55.

120. Belyanina, I.; Kolovskaya, O.; Zamay, S.; Gargaun, A.; Zamay, T.; Kichkailo, A., Targeted Magnetic Nanotheranostics of Cancer. Molecules 2017, 22 (6), 975.

121. Wang, C.; Ravi, S.; Garapati, U. S.; Das, M.; Howell, M.; MallelaMallela, J.; Alwarapan, S.; Mohapatra, S. S.; Mohapatra, S., Multifunctional Chitosan Magnetic-Graphene (CMG) Nanoparticles: a Theranostic Platform for Tumor-targeted Co-delivery of Drugs, Genes and MRI Contrast Agents. Journal of materials chemistry. B 2013, 1 (35), 4396-4405.

122. Jose, J.; Kumar, R.; Harilal, S.; Mathew, G. E.; Parambi, D. G. T.; Prabhu, A.; Uddin, M. S.; Aleya, L.; Kim, H.; Mathew, B., Magnetic nanoparticles for hyperthermia in cancer treatment: an emerging tool. Environmental science and pollution research international 2020, 27 (16), 19214-19225.

123. Legge, C. J.; Colley, H. E.; Lawson, M. A.; Rawlings, A. E., Targeted magnetic nanoparticle hyperthermia for the treatment of oral cancer. Journal of oral pathology \& medicine : official publication of the International Association of Oral Pathologists and the American Academy of Oral Pathology 2019, 48 (9), 803-809.

124. Majeed, J.; Pradhan, L.; Ningthoujam, R. S.; Vatsa, R. K.; Bahadur, D.; Tyagi, A. K., Enhanced specific absorption rate in silanol functionalized Fe3O4 core-shell nanoparticles: study of Fe leaching in Fe3O4 and hyperthermia in L929 and HeLa cells. Colloids and surfaces. B, Biointerfaces 2014, 122, 396403.

125. Mondal, S.; Manivasagan, P.; Bharathiraja, S.; Santha Moorthy, M.; Nguyen, V. T.; Kim, H. H.; Nam, S. Y.; Lee, K. D.; Oh, J., Hydroxyapatite Coated Iron Oxide Nanoparticles: A Promising Nanomaterial for Magnetic Hyperthermia Cancer Treatment. Nanomaterials 2017, 7 (12), 426.

126. Jang, J. T.; Lee, J.; Seon, J.; Ju, E.; Kim, M.; Kim, Y. I.; Kim, M. G.; Takemura, Y.; Arbab, A. S.; Kang, K. W.; Park, K. H.; Paek, S. H.; Bae, S., Giant Magnetic Heat Induction of Magnesium-Doped gamma-Fe2 O3 Superparamagnetic Nanoparticles for Completely Killing Tumors. Adv Mater 2018, 30 (6), 1704362.

127. Hedayatnasab, Z.; Dabbagh, A.; Abnisa, F.; Wan Daud, W. M. A., Polycaprolactone-coated superparamagnetic iron oxide nanoparticles for in vitro magnetic hyperthermia therapy of cancer. European Polymer Journal 2020, 133, 109789.

128. Khizar, S.; Ahmad, N. M.; Saleem, H.; Hamayun, M. A.; Manzoor, S.; Lebaz, N.; Elaissari, A., Magnetic Colloidal Particles in Combinatorial Thin-Film Gradients for Magnetic Resonance Imaging and Hyperthermia. Advances in Polymer Technology 2020, 2020, 1-18.

129. Wang, H.; Zhang, X., Magnetic Fields and Reactive Oxygen Species. International journal of molecular sciences 2017, 18 (10), 2175.

130. Chen, L.; Chen, C.; Wang, P.; Song, T., Mechanisms of Cellular Effects Directly Induced by Magnetic Nanoparticles under Magnetic Fields. Journal of Nanomaterials 2017, 2017, 1-13.

131. Sola-Leyva, A.; Jabalera, Y.; Chico-Lozano, M. A.; Carrasco-Jimenez, M. P.; Iglesias, G. R.; Jimenez-Lopez, C., Reactive oxygen species (ROS) production in HepG2 cancer cell line through the application of localized alternating magnetic field. Journal of materials chemistry. B 2020, 8 (34), 76677676.

132. Johannsen, M.; Thiesen, B.; Wust, P.; Jordan, A., Magnetic nanoparticle hyperthermia for prostate cancer. International journal of hyperthermia : the official journal of European Society for Hyperthermic Oncology, North American Hyperthermia Group 2010, 26 (8), 790-5.

133. Destouches, D.; Page, N.; Hamma-Kourbali, Y.; Machi, V.; Chaloin, O.; Frechault, S.; Birmpas, C.; Katsoris, P.; Beyrath, J.; Albanese, P.; Maurer, M.; Carpentier, G.; Strub, J. M.; Van Dorsselaer, A.; Muller, 
S.; Bagnard, D.; Briand, J. P.; Courty, J., A simple approach to cancer therapy afforded by multivalent pseudopeptides that target cell-surface nucleoproteins. Cancer research 2011, 71 (9), 3296-305.

134. Maier-Hauff, K.; Ulrich, F.; Nestler, D.; Niehoff, H.; Wust, P.; Thiesen, B.; Orawa, H.; Budach, V.; Jordan, A., Efficacy and safety of intratumoral thermotherapy using magnetic iron-oxide nanoparticles combined with external beam radiotherapy on patients with recurrent glioblastoma multiforme. Journal of neuro-oncology 2011, 103 (2), 317-24.

135. Matsumine, A.; Takegami, K.; Asanuma, K.; Matsubara, T.; Nakamura, T.; Uchida, A.; Sudo, A., A novel hyperthermia treatment for bone metastases using magnetic materials. International journal of clinical oncology 2011, 16 (2), 101-8.

136. Alomari, M.; Almohazey, D.; Almofty, S.; Alhibshi, A.; Almansour, I.; Kaewsaneha, C.; Badri, W.; Fessi, H.; Elaissari, A., Magnetic-responsive polysaccharide-inorganic composite materials for cancer therapeutics. 2019, 179-216.

137. Chertok, B.; David, A. E.; Yang, V. C., Polyethyleneimine-modified iron oxide nanoparticles for brain tumor drug delivery using magnetic targeting and intra-carotid administration. Biomaterials 2010, 31 (24), 6317-24.

138. Chen, F. H.; Zhang, L. M.; Chen, Q. T.; Zhang, Y.; Zhang, Z. J., Synthesis of a novel magnetic drug delivery system composed of doxorubicin-conjugated $\mathrm{Fe} 3 \mathrm{O} 4$ nanoparticle cores and a PEGfunctionalized porous silica shell. Chemical communications 2010, 46 (45), 8633-5.

139. Linares, V.; Yarce, C. J.; Echeverri, J. D.; Galeano, E.; Salamanca, C. H., Relationship between Degree of Polymeric lonisation and Hydrolytic Degradation of Eudragit((R)) E Polymers under Extreme Acid Conditions. Polymers (Basel) 2019, 11 (6), 1010.

140. Jordan, A.; Scholz, R.; Maier-Hauff, K.; van Landeghem, F. K.; Waldoefner, N.; Teichgraeber, U.; Pinkernelle, J.; Bruhn, H.; Neumann, F.; Thiesen, B.; von Deimling, A.; Felix, R., The effect of thermotherapy using magnetic nanoparticles on rat malignant glioma. Journal of neuro-oncology 2006, 78 (1), 7-14.

141. Zhao, Q.; Wang, L.; Cheng, R.; Mao, L.; Arnold, R. D.; Howerth, E. W.; Chen, Z. G.; Platt, S., Magnetic nanoparticle-based hyperthermia for head \& neck cancer in mouse models. Theranostics 2012, 2, 113-21.

142. Nandwana, V.; De, M.; Chu, S.; Jaiswal, M.; Rotz, M.; Meade, T. J.; Dravid, V. P., Theranostic Magnetic Nanostructures (MNS) for Cancer. Cancer treatment and research 2015, 166, 51-83.

143. Sirivisoot, S.; Harrison, B. S., Magnetically stimulated ciprofloxacin release from polymeric microspheres entrapping iron oxide nanoparticles. International journal of nanomedicine 2015, 10 , 4447-58.

144. Jia, Y.; Yuan, M.; Yuan, H.; Huang, X.; Sui, X.; Cui, X.; Tang, F.; Peng, J.; Chen, J.; Lu, S.; Xu, W.; Zhang, L.; Guo, Q., Co-encapsulation of magnetic Fe3O4 nanoparticles and doxorubicin into biodegradable PLGA nanocarriers for intratumoral drug delivery. International journal of nanomedicine 2012, 7, 1697-708.

145. Niu, C.; Wang, Z.; Lu, G.; Krupka, T. M.; Sun, Y.; You, Y.; Song, W.; Ran, H.; Li, P.; Zheng, Y., Doxorubicin loaded superparamagnetic PLGA-iron oxide multifunctional microbubbles for dual-mode US/MR imaging and therapy of metastasis in lymph nodes. Biomaterials 2013, 34 (9), 2307-17.

146. Schleich, N.; Sibret, P.; Danhier, P.; Ucakar, B.; Laurent, S.; Muller, R. N.; Jerome, C.; Gallez, B.; Preat, V.; Danhier, F., Dual anticancer drug/superparamagnetic iron oxide-loaded PLGA-based nanoparticles for cancer therapy and magnetic resonance imaging. International journal of pharmaceutics 2013, 447 (1-2), 94-101.

147. Akbarzadeh, A.; Mikaeili, H.; Zarghami, N.; Mohammad, R.; Barkhordari, A.; Davaran, S., Preparation and in vitro evaluation of doxorubicin-loaded $\mathrm{Fe}(3) \mathrm{O}(4)$ magnetic nanoparticles modified with biocompatible copolymers. International journal of nanomedicine 2012, 7, 511-26. 
148. Asadi, N.; Annabi, N.; Mostafavi, E.; Anzabi, M.; Khalilov, R.; Saghfi, S.; Mehrizadeh, M.; Akbarzadeh, A., Synthesis, characterization and in vitro evaluation of magnetic nanoparticles modified with PCL-PEG-PCL for controlled delivery of 5FU. Artificial cells, nanomedicine, and biotechnology 2018, 46 (sup1), 938-945.

149. Mosafer, J.; Abnous, K.; Tafaghodi, M.; Jafarzadeh, H.; Ramezani, M., Preparation and characterization of uniform-sized PLGA nanospheres encapsulated with oleic acid-coated magnetic-Fe 3 O 4 nanoparticles for simultaneous diagnostic and therapeutic applications. Colloids and Surfaces A: Physicochemical and Engineering Aspects 2017, 514, 146-154.

150. Ayubi, M.; Karimi, M.; Abdpour, S.; Rostamizadeh, K.; Parsa, M.; Zamani, M.; Saedi, A., Magnetic nanoparticles decorated with PEGylated curcumin as dual targeted drug delivery: Synthesis, toxicity and biocompatibility study. Materials science \& engineering. C, Materials for biological applications 2019, 104, 109810.

151. Attari, E.; Nosrati, H.; Danafar, H.; Kheiri Manjili, H., Methotrexate anticancer drug delivery to breast cancer cell lines by iron oxide magnetic based nanocarrier. Journal of biomedical materials research. Part A 2019, 107 (11), 2492-2500.

152. Karimi Ghezeli, Z.; Hekmati, M.; Veisi, H., Synthesis of Imatinib-loaded chitosan-modified magnetic nanoparticles as an anti-cancer agent for $\mathrm{pH}$ responsive targeted drug delivery. Applied Organometallic Chemistry 2019, 33 (4), e4833.

153. Dey, C.; Das, A.; Goswami, M. M., Dopamine Loaded SiO2 Coated Fe3O4 Magnetic Nanoparticles: A New Anticancer Agent in pH-Dependent Drug Delivery. ChemistrySelect 2019, 4 (41), 12190-12196.

154. Patil, A. R.; Nimbalkar, M. S.; Patil, P. S.; Chougale, A. D.; Patil, P. B., Controlled release of poorly water soluble anticancerous drug camptothecin from magnetic nanoparticles. Materials Today: Proceedings 2020, 23, 437-443.

155. Keyvan Rad, J.; Alinejad, Z.; Khoei, S.; Mahdavian, A. R., Controlled Release and Photothermal Behavior of Multipurpose Nanocomposite Particles Containing Encapsulated Gold-Decorated Magnetite and 5-FU in Poly(lactide-co-glycolide). ACS Biomater Sci Eng 2019, 5 (9), 4425-4434.

156. Linsley, C. S.; Wu, B. M., Recent advances in light-responsive on-demand drug-delivery systems. Therapeutic delivery 2017, 8 (2), 89-107.

157. Sahle, F. F.; Gulfam, M.; Lowe, T. L., Design strategies for physical-stimuli-responsive programmable nanotherapeutics. Drug discovery today 2018, 23 (5), 992-1006.

158. Lalegul-Ulker, O.; Elcin, Y. M., Magnetic and electrically conductive silica-coated iron oxide/polyaniline nanocomposites for biomedical applications. Materials science \& engineering. $C$, Materials for biological applications 2021, 119, 111600.

159. Reyes-Ortega, F.; Delgado, A. V.; Schneider, E. K.; Checa Fernandez, B. L.; Iglesias, G. R., Magnetic Nanoparticles Coated with a Thermosensitive Polymer with Hyperthermia Properties. Polymers (Basel) 2017, 10 (1), 10.

160. Chircov, C.; Grumezescu, A. M.; Holban, A. M., Magnetic Particles for Advanced Molecular Diagnosis. Materials 2019, 12 (13), 2158.

161. Hola, K.; Markova, Z.; Zoppellaro, G.; Tucek, J.; Zboril, R., Tailored functionalization of iron oxide nanoparticles for MRI, drug delivery, magnetic separation and immobilization of biosubstances. Biotechnology advances 2015, 33 (6 Pt 2), 1162-76.

162. Nash, M. A.; Yager, P.; Hoffman, A. S.; Stayton, P. S., Mixed stimuli-responsive magnetic and gold nanoparticle system for rapid purification, enrichment, and detection of biomarkers. Bioconjugate chemistry 2010, 21 (12), 2197-204.

163. Mettler, E.; Trenkler, A.; Feilen, P. J.; Wiegand, F.; Fottner, C.; Ehrhart, F.; Zimmermann, H.; Hwang, Y. H.; Lee, D. Y.; Fischer, S.; Schreiber, L. M.; Weber, M. M., Magnetic separation of encapsulated 
islet cells labeled with superparamagnetic iron oxide nano particles. Xenotransplantation 2013, 20 (4), 219-26.

164. Lui, C. N. P.; Tsui, Y. P.; Ho, A. S. L.; Shum, D. K. Y.; Chan, Y. S.; Wu, C. T.; Li, H. W.; Tsang, S. C. E.; Yung, K. K. L., Neural Stem Cells Harvested from Live Brains by Antibody-Conjugated Magnetic Nanoparticles. Angewandte Chemie 2013, 125 (47), 12524-12528.

165. Holschuh, K.; Schwämmle, A., Preparative purification of antibodies with protein A-an alternative to conventional chromatography. Journal of Magnetism and Magnetic Materials 2005, 293 (1), 345-348.

166. Rahman, M. M.; Elaissari, A., Temperature and magnetic dual responsive microparticles for DNA separation. Separation and Purification Technology 2011, 81 (3), 286-294.

167. Patra, J. K.; Das, G.; Fraceto, L. F.; Campos, E. V. R.; Rodriguez-Torres, M. D. P.; Acosta-Torres, L. S.; Diaz-Torres, L. A.; Grillo, R.; Swamy, M. K.; Sharma, S.; Habtemariam, S.; Shin, H. S., Nano based drug delivery systems: recent developments and future prospects. Journal of nanobiotechnology 2018, 16 (1), 71.

168. Kadian, R., Nanoparticles: A Promising Drug Delivery Approach. Asian Journal of Pharmaceutical and Clinical Research 2018, 11 (1), 30.

169. Taratula, O.; Dani, R. K.; Schumann, C.; Xu, H.; Wang, A.; Song, H.; Dhagat, P.; Taratula, O., Multifunctional nanomedicine platform for concurrent delivery of chemotherapeutic drugs and mild hyperthermia to ovarian cancer cells. International journal of pharmaceutics 2013, 458 (1), 169-80.

170. Xue, W.; Liu, X. L.; Ma, H.; Xie, W.; Huang, S.; Wen, H.; Jing, G.; Zhao, L.; Liang, X. J.; Fan, H. M., AMF responsive DOX-loaded magnetic microspheres: transmembrane drug release mechanism and multimodality postsurgical treatment of breast cancer. Journal of materials chemistry. B 2018, 6 (15), 2289-2303.

171. Zhu, X.; Zhang, H.; Huang, H.; Zhang, Y.; Hou, L.; Zhang, Z., Functionalized graphene oxide-based thermosensitive hydrogel for magnetic hyperthermia therapy on tumors. Nanotechnology 2015, 26 (36), 365103.

172. Fang, K.; Song, L.; Gu, Z.; Yang, F.; Zhang, Y.; Gu, N., Magnetic field activated drug release system based on magnetic PLGA microspheres for chemo-thermal therapy. Colloids and surfaces. $B$, Biointerfaces 2015, 136, 712-20.

173. Cho, H. Y.; Lee, T.; Yoon, J.; Han, Z.; Rabie, H.; Lee, K. B.; Su, W. W.; Choi, J. W., Magnetic Oleosome as a Functional Lipophilic Drug Carrier for Cancer Therapy. ACS applied materials \& interfaces 2018, 10 (11), 9301-9309.

174. Sanhaji, M.; Goring, J.; Couleaud, P.; Aires, A.; Cortajarena, A. L.; Courty, J.; Prina-Mello, A.; Stapf, M.; Ludwig, R.; Volkov, Y.; Latorre, A.; Somoza, A.; Miranda, R.; Hilger, I., The phenotype of target pancreatic cancer cells influences cell death by magnetic hyperthermia with nanoparticles carrying gemicitabine and the pseudo-peptide NucAnt. Nanomedicine : nanotechnology, biology, and medicine 2019, 20, 101983.

175. Liu, D.; Hong, Y.; Li, Y.; Hu, C.; Yip, T. C.; Yu, W. K.; Zhu, Y.; Fong, C. C.; Wang, W.; Au, S. K.; Wang, S.; Yang, M., Targeted destruction of cancer stem cells using multifunctional magnetic nanoparticles that enable combined hyperthermia and chemotherapy. Theranostics 2020, 10 (3), 1181-1196.

176. Gao, F.; Xie, W.; Miao, Y.; Wang, D.; Guo, Z.; Ghosal, A.; Li, Y.; Wei, Y.; Feng, S. S.; Zhao, L.; Fan, H. M., Magnetic Hydrogel with Optimally Adaptive Functions for Breast Cancer Recurrence Prevention. Advanced healthcare materials 2019, 8 (14), e1900203.

177. Ha, P. T.; Le, T. T. H.; Bui, T. Q.; Pham, H. N.; Ho, A. S.; Nguyen, L. T., Doxorubicin release by magnetic inductive heating and in vivo hyperthermia-chemotherapy combined cancer treatment of multifunctional magnetic nanoparticles. New Journal of Chemistry 2019, 43 (14), 5404-5413.

178. Mohammed, L.; Gomaa, H. G.; Ragab, D.; Zhu, J., Magnetic nanoparticles for environmental and biomedical applications: A review. Particuology 2017, 30, 1-14. 
179. Dizaj, S. M.; Jafari, S.; Khosroushahi, A. Y., A sight on the current nanoparticle-based gene delivery vectors. Nanoscale research letters 2014, 9 (1), 252.

180. Majidi, S.; Zeinali Sehrig, F.; Samiei, M.; Milani, M.; Abbasi, E.; Dadashzadeh, K.; Akbarzadeh, A., Magnetic nanoparticles: Applications in gene delivery and gene therapy. Artificial cells, nanomedicine, and biotechnology 2016, 44 (4), 1186-93.

181. Magro, M.; Martinello, T.; Bonaiuto, E.; Gomiero, C.; Baratella, D.; Zoppellaro, G.; Cozza, G.; Patruno, M.; Zboril, R.; Vianello, F., Covalently bound DNA on naked iron oxide nanoparticles: Intelligent colloidal nano-vector for cell transfection. Biochimica et biophysica acta. General subjects 2017, 1861 (11 Pt A), 2802-2810.

182. Morishita, N.; Nakagami, H.; Morishita, R.; Takeda, S.; Mishima, F.; Terazono, B.; Nishijima, S.; Kaneda, Y.; Tanaka, N., Magnetic nanoparticles with surface modification enhanced gene delivery of HVJ-E vector. Biochemical and biophysical research communications 2005, 334 (4), 1121-6.

183. Zhao, X.; Cui, H.; Chen, W.; Wang, Y.; Cui, B.; Sun, C.; Meng, Z.; Liu, G., Morphology, structure and function characterization of PEI modified magnetic nanoparticles gene delivery system. PloS one 2014, 9 (6), e98919.

184. Uthaman, S.; Lee, S. J.; Cherukula, K.; Cho, C. S.; Park, I. K., Polysaccharide-Coated Magnetic Nanoparticles for Imaging and Gene Therapy. BioMed research international 2015, 2015, 959175.

185. Mu, X.; Qiao, J.; Qi, L.; Dong, P.; Ma, H., Poly(2-vinyl-4,4-dimethylazlactone)-functionalized magnetic nanoparticles as carriers for enzyme immobilization and its application. ACS applied materials \& interfaces 2014, 6 (23), 21346-54.

186. Kumar, S.; Jana, A. K.; Dhamija, I.; Maiti, M., Chitosan-assisted immobilization of serratiopeptidase on magnetic nanoparticles, characterization and its target delivery. Journal of drug targeting 2014, 22 (2), 123-37.

187. Wang, J.; Meng, G.; Tao, K.; Feng, M.; Zhao, X.; Li, Z.; Xu, H.; Xia, D.; Lu, J. R., Immobilization of lipases on alkyl silane modified magnetic nanoparticles: effect of alkyl chain length on enzyme activity. PloS one 2012, 7 (8), e43478.

188. Atacan, K.; Ozacar, M., Characterization and immobilization of trypsin on tannic acid modified Fe3O4 nanoparticles. Colloids and surfaces. B, Biointerfaces 2015, 128, 227-236.

189. Xu, J.; Sun, J.; Wang, Y.; Sheng, J.; Wang, F.; Sun, M., Application of iron magnetic nanoparticles in protein immobilization. Molecules 2014, 19 (8), 11465-86.

190. Revia, R. A.; Zhang, M., Magnetite nanoparticles for cancer diagnosis, treatment, and treatment monitoring: recent advances. Mater Today (Kidlington) 2016, 19 (3), 157-168.

191. Estelrich, J.; Busquets, M. A., Iron Oxide Nanoparticles in Photothermal Therapy. Molecules 2018, 23 (7), 1567.

192. Lu, J. W.; Yang, F.; Ke, Q. F.; Xie, X. T.; Guo, Y. P., Magnetic nanoparticles modified-porous scaffolds for bone regeneration and photothermal therapy against tumors. Nanomedicine : nanotechnology, biology, and medicine 2018, 14 (3), 811-822.

193. Bu, L. L.; Rao, L.; Yu, G. T.; Chen, L.; Deng, W. W.; Liu, J. F.; Wu, H.; Meng, Q. F.; Guo, S. S.; Zhao, X. Z.; Zhang, W. F.; Chen, G.; Gu, Z.; Liu, W.; Sun, Z. J., Cancer Stem Cell-Platelet Hybrid MembraneCoated Magnetic Nanoparticles for Enhanced Photothermal Therapy of Head and Neck Squamous Cell Carcinoma. Advanced functional materials 2019, 29 (10), 1807733.

194. Lu, Q.; Dai, X.; Zhang, P.; Tan, X.; Zhong, Y.; Yao, C.; Song, M.; Song, G.; Zhang, Z.; Peng, G.; Guo, Z.; Ge, Y.; Zhang, K.; Li, Y., Fe304@Au composite magnetic nanoparticles modified with cetuximab for targeted magneto-photothermal therapy of glioma cells. International journal of nanomedicine 2018, 13, 2491-2505.

195. Ma, K.; Liu, G.-J.; Yan, L.; Wen, S.; Xu, B.; Tian, W.; Goldys, E. M.; Liu, G., AlEgen based poly(Llactic-co-glycolic acid) magnetic nanoparticles to localize cytokine VEGF for early cancer diagnosis and photothermal therapy. Nanomedicine : nanotechnology, biology, and medicine 2019, 14 (9), 1191-1201. 
196. Zhang, F.; Lu, G.; Wen, X.; Li, F.; Ji, X.; Li, Q.; Wu, M.; Cheng, Q.; Yu, Y.; Tang, J.; Mei, L., Magnetic nanoparticles coated with polyphenols for spatio-temporally controlled cancer photothermal/immunotherapy. Journal of controlled release : official journal of the Controlled Release Society 2020, 326, 131-139.

197. Jedrzak, A.; Grzeskowiak, B. F.; Coy, E.; Wojnarowicz, J.; Szutkowski, K.; Jurga, S.; Jesionowski, T.; Mrowczynski, R., Dendrimer based theranostic nanostructures for combined chemo- and photothermal therapy of liver cancer cells in vitro. Colloids and surfaces. B, Biointerfaces 2019, 173, 698-708.

198. Wu, W.; Jiang, C. Z.; Roy, V. A., Designed synthesis and surface engineering strategies of magnetic iron oxide nanoparticles for biomedical applications. Nanoscale 2016, 8 (47), 19421-19474.

199. Kheshtzar, R.; Berenjian, A.; Taghizadeh, S.-M.; Ghasemi, Y.; Asad, A. G.; Ebrahiminezhad, A., Optimization of reaction parameters for the green synthesis of zero valent iron nanoparticles using pine tree needles. Green Processing and Synthesis 2019, 8 (1), 846-855.

200. Long, N. V.; Yang, Y.; Teranishi, T.; Thi, C. M.; Cao, Y.; Nogami, M., Biomedical Applications of Advanced Multifunctional Magnetic Nanoparticles. Journal of nanoscience and nanotechnology 2015, 15 (12), 10091-107.

201. Mitchell, M. J.; Billingsley, M. M.; Haley, R. M.; Wechsler, M. E.; Peppas, N. A.; Langer, R., Engineering precision nanoparticles for drug delivery. Nature reviews. Drug discovery 2021, 20 (2), 101124.

202. Saadat, M.; Manshadi, M. K. D.; Mohammadi, M.; Zare, M. J.; Zarei, M.; Kamali, R.; SanatiNezhad, A., Magnetic particle targeting for diagnosis and therapy of lung cancers. Journal of controlled release : official journal of the Controlled Release Society 2020, 328, 776-791.

203. Fan, D.; Wang, Q.; Zhu, T.; Wang, H.; Liu, B.; Wang, Y.; Liu, Z.; Liu, X.; Fan, D.; Wang, X., Recent Advances of Magnetic Nanomaterials in Bone Tissue Repair. Frontiers in chemistry 2020, 8, 745.

204. Arias, L. S.; Pessan, J. P.; Vieira, A. P. M.; Lima, T. M. T.; Delbem, A. C. B.; Monteiro, D. R., Iron Oxide Nanoparticles for Biomedical Applications: A Perspective on Synthesis, Drugs, Antimicrobial Activity, and Toxicity. Antibiotics 2018, 7 (2), 46.

205. Doswald, S.; Stark, W. J.; Beck-Schimmer, B., Biochemical functionality of magnetic particles as nanosensors: how far away are we to implement them into clinical practice? Journal of nanobiotechnology 2019, 17 (1), 73. 


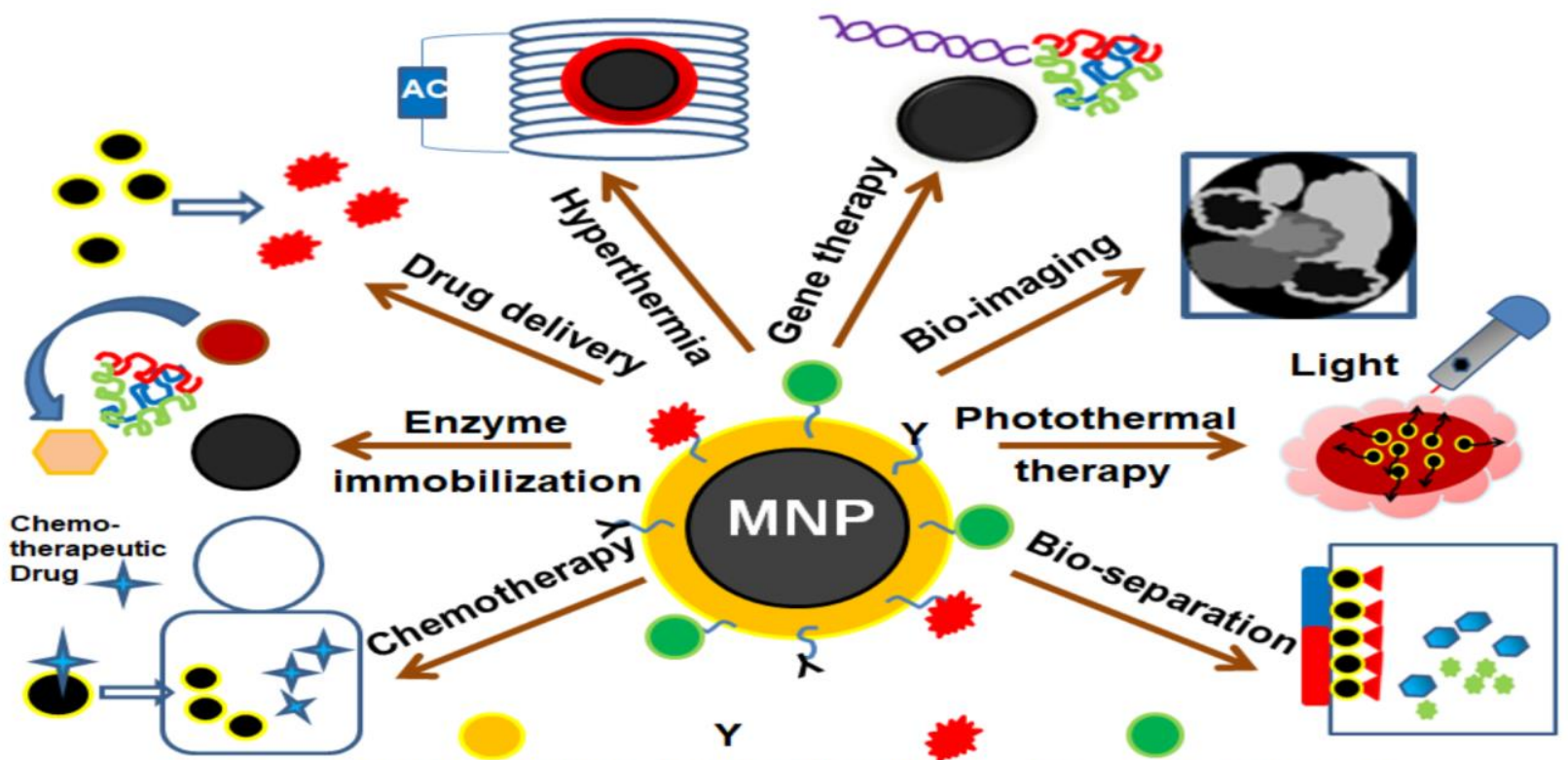

Coating Layer Antibodies Drug Targeting Ligands 